\title{
FvSTUA is a Key Regulator of Sporulation, Toxin Synthesis, and Virulence in Fusarium verticillioides
}

\author{
M. Rath, ${ }^{1}$ N. J. Crenshaw, ${ }^{2}$ L. W. Lofton, ${ }^{2}$ A. E. Glenn, ${ }^{2}$ and S. E. Gold ${ }^{2,+}$ \\ ${ }^{1}$ Department of Plant Pathology, University of Georgia, Athens, GA, U.S.A. \\ ${ }^{2}$ USDA, ARS, US National Poultry Research Center, Toxicology \& Mycotoxin Research Unit, Athens, GA, U.S.A.
}

Accepted 9 April 2020.

\begin{abstract}
Fusarium verticillioides is one of the most important pathogens of maize, causing rot and producing fumonisin mycotoxins during infection. Ingestion of fumonisin-contaminated corn causes underperformance and even fatal toxicity in livestock and is associated with neural tube birth defects, growth stunting in children, and some cancers. StuA, an APSES-class transcription factor, is a major developmental transcriptional regulator in fungi. It has been shown to regulate crucial developmental processes, such as sporulation, virulence, and mycotoxin synthesis among others. In this study, the role of FvSTUA in $F$. verticillioides was examined by characterizing $\Delta F v s t u A$ deletion mutants functionally and transcriptomally. The deletion mutants exhibited reduced vegetative growth, stunted aerial hyphae, and significant reductions in microconidiation. Macroconidiation and hydrophobicity of the deletion strains were reduced as well. Additionally, fumonisin production and virulence of the deletion mutants were greatly reduced. Transcriptomic analysis revealed downregulation of expression of several genes in the fumonisin and fusarin $\mathbf{C}$ biosynthetic clusters and differential expression of genes involved in conidiation and virulence. Nuclear localization of FvSTUA supported its likely function as a transcription factor. Together, our results indicate that FvSTUA plays a global role in transcriptional regulation in $F$. verticillioides influencing morphogenesis, toxin production, and virulence.
\end{abstract}

Keywords: fungus-plant interactions, Fusarium verticillioides, fungal development, fumonisin, maize, mechanisms of pathogenicity, mycotoxins

Fusarium verticillioides, a member of the phylum Ascomycota, is one of the most ubiquitous and devastating pathogens of maize. It is prevalent on field cultivars in current use (Bacon et al. 2008; Shephard et al. 1996). On maize, $F$. verticillioides functions as a hemibiotrophic pathogen or an

${ }^{\dagger}$ Corresponding author: S. Gold; scott.gold@usda.gov

Funding: This work was supported by the US Department of Agriculture, Agricultural Research Service (USDA-ARS) project number 6040-42000043-00D.

*The $\boldsymbol{e}$-Xtra logo stands for "electronic extra" and indicates that supplementary material is published online.

The author(s) declare no conflict of interest.

This article is in the public domain and not copyrightable. It may be freely reprinted with customary crediting of the source. The American Phytopathological Society, 2020. endophyte (Bacon and Hinton 1996; Bacon et al. 2001, 2008; White 1999). The pathogen causes root, stalk, and ear rot in maize (Foley 1962) and, importantly, produces the fumonisin family of mycotoxins (Bacon et al. 2008; Marasas 2001; Munkvold and Desjardins 1997; Pitt et al. 2012). Fumonisin B1 $\left(\mathrm{FB}_{1}\right)$ is the most abundant and toxic fumonisin produced in maize (Musser and Plattner 1997), and ingesting $\mathrm{FB}_{1}$-contaminated corn has been associated with high animal toxicity (e.g., equine leukoencephalomalacia and porcine pulmonary edema) (Abel and Gelderblom 1998). In humans, fumonisin has been associated with esophageal cancer, neural tube birth defects, and stunted growth of children (Marasas 2001; Rheeder et al. 1992). Fumonisins are synthesized by $F$. verticillioides, throughout its association with maize, as a consequence of tissue damage or stress. Fumonisins can also be synthesized in the endophytic state (Bacon et al. 2001; Gatch and Munkvold 2002; Munkvold et al. 1997). Besides the human and animal health risks due to toxin exposure, $F$. verticillioides-infected and fumonisincontaminated grain may be rejected in the marketplace, resulting in losses in the tens of millions of dollars per year (Wu 2004, 2006).

The primary source of inoculum for $F$. verticillioides is asexually produced conidia, which can infect the host plant through root and shoot tissues (Desjardins 2003; Leslie and Summerell 2006; Yates et al. 2003). Additionally, F. verticillioides typically produces two types of conidia, single-celled microconidia, which are the most abundant asexual spore form in this species, and larger, septate macroconidia, which are usually much less abundant (Leslie and Summerell 2006; Nelson et al. 1983). Several genes (e.g., HYD1 and HYD2, FCC1, FPH1 and $F v V E 1$ ) have been reported to be involved in the morphogenesis of F. verticillioides (Fuchs et al. 2004; Glenn 2006; $\mathrm{Li}$ et al. 2006; Shim and Woloshuk 2001). In some studies, as with other mycotoxigenic fungi, fumonisin production in $F$. verticillioides is developmentally and genetically coordinated with asexual conidiation (Choi and Shim 2008; Glenn et al. 2004; Sagaram et al. 2007; Shim and Woloshuk 2001; Shin et al. 2013).

The APSES (ASM-1, Phd1, StuA, EFG1, and Sok2) protein family is comprised of transcription factors that regulate various biological processes such as conidiophore morphogenesis, vegetative growth, dimorphic switching, secondary metabolism, and virulence in fungi (Aramayo et al. 1996; Gimeno and Fink 1994; Miller et al. 1991; Stoldt et al. 1997; Twumasi-Boateng et al. 2009; Ward et al. 1995; Zhao et al. 2015). These proteins possess the APSES domain, a basic helix-loop-helix (bHLH) region, that incorporates one or more copies of the highly conserved DNA-binding domain. StuA, an APSES-class transcription factor first discovered in Aspergillus nidulans, was found to be a key regulator of developmental and cell-cycle genes (Dutton 
et al. 1997; Miller et al. 1992; Wu and Miller 1997). Since then, StuA orthologs have been characterized in several phytopathogenic fungi, such as Magnaporthe oryzae (MStu1), Ustilago maydis (Ust1), Glomerella cingulata (GcStuA), Stagonospora nodorum (SnStuA), and has been shown to impact essential biological processes such as sporulation, secondary metabolite production, mycelial growth, and virulence in these species (Baeza-Montañez et al. 2015; García-Pedrajas et al. 2010; IpCho et al. 2010; Islamovic et al. 2015; Nishimura et al. 2009; Tong et al. 2007). In Fusarium graminearum, FgStuA acts as a global regulator influencing processes such as toxin (deoxynivalenol $[\mathrm{DON}])$ production, conidiation, virulence and pathogenicity (Lysøe et al. 2011). On the other hand, studies on FoStuA in Fusarium oxysporum f. sp. melonis showed no effect on pathogenicity, although it did regulate development of asexual conidiation (Ohara and Tsuge 2004). A study on conidiation regulatory genes in $F$. verticillioides indicated that mutation of FvSTUA negatively impacted microconidiation (Malapi-Wight et al. 2014). In light of the discovery of the role of StuA in pathogenicity and secondary metabolism of several fungal plant pathogens, documenting the roles of FvSTUA in $F$. verticillioides could lead to important information regarding a number of developmental and biological processes in this plant pathogen, particularly in terms of its potential regulation of fumonisin mycotoxin production.

We investigated the function of FvSTUA in F. verticillioides by characterizing $\Delta F v s t u A$ deletion strains. Our results revealed that FvSTUA is important for radial mycelial growth, conidiation, virulence, and fumonisin biosynthesis in F. verticillioides, similar to other StuA homologs. Nuclear localization of FvSTUA was demonstrated, consistent with its function as a transcription factor. Transcriptomic analysis revealed that FvSTUA regulates synthesis of fumonisins, fusarin $\mathrm{C}$, and fusaric acid and affects conidiation- and pathogencity-associated genes. Our results provide evidence that FVSTUA is a major regulator of morphological differentiation, virulence, and toxin biosynthesis in $F$. verticillioides.

\section{RESULTS}

In silico characterization of $\boldsymbol{F}$. verticillioides FvStuA.

Malapi-Wight et al. (2014) identified the gene FvSTUA (FVEG_02853) as encoding a putative APSES class transcription factor and a homolog to StuA of Aspergillus nidulans. Here, further characterization of FvStuA indicated that the predicted primary transcript is $3,470 \mathrm{bp}$ in length with four exons with a predicted mature transcript length of 3,100 bp. The predicted protein sequence is 549 amino acids (aa) in length, with a mass of $59.65 \mathrm{kDa}$ and isoelectric point of 8.57 . A Uniprot database search revealed that the predicted FvSTUA protein consists of the conserved APSES-type HTH DNAbinding domain (IPR003163) 125 aa in length (from position 87 to 212 aa) (Fig. 1). The InterPro database identified an additional KilA, N-terminal/APSES-type HTH domain (IPR018004) assigned to FvSTUA between aa position 106-191. National Center for Biotechnology Information BLAST of the predicted FvSTUA protein sequence showed homology with putative and confirmed StuA proteins of a number of Fusarium spp., including F. proliferatum, F. fujikuroi, and F. graminearum, as well as StuA proteins of other diverse Ascomycota, such as Magnaporthe oryzae, Blumeria graminis, Verticillium dahliae, Colletotrichum graminicola, and Gaeumannomyces tritici. FvSTUA showed the highest homology with the StuA of Fusarium oxysporum f. sp. cubense race $4(98 \%$ identity and $\mathrm{E}$ value $=0.0)$. The high level of sequence similarity of the putative FvSTUA protein with StuA homologs in other Fusarium spp. indicated that FvSTUA is indeed the $F$. verticillioides StuA homolog. Figure 1 shows the domain conservation and positioning of FvStuA with respect to those of the canonical APSES proteins and to other Fusarium species.

\section{Intracellular and nuclear localization of FvSTUA-tdTomato.}

Using WoLFPSORT at FungiDB, 21 of 27 "nearest neighbors" indicated nuclear localization, consistent with its role as a transcription factor (data not shown). A nuclear localization domain was identified near the C-terminus of the protein (from position 516-545 aa), via Uniprot search (not shown). To experimentally test the intracellular localization of FvSTUA, we constructed plasmid pFvSTUA-tdTomato for expressing the fusion protein FvSTUA-tdTomato. The fusion gene was expressed under the control of the native FvSTUA promoter. After introducing the FvSTUA-tdTomato plasmid into $F$. verticillioides wild-type (WT) strain M3125 through Agrobacterium-mediated transformation (ATMT), transformants were randomly selected and the intracellular distribution of the tdTomato fluorescence was observed using fluorescence microscopy. Our results revealed that, together with occasional diffuse cytoplasmic fluorescence, FvSTUA clearly localizes to nuclei of $F$. verticillioides (Fig. 2). This result is consistent with the prediction that FvSTUA functions as a transcriptional regulator.

\section{Generation and confirmation}

of $\Delta F v s t u A$ deletion transformants and progeny.

ATMT of the WT strain resulted in the identification of six $\Delta F v s t u A$ mutants $(\Delta F v s t u A 1 / 2, \Delta F v s t u A 1 / 3, \Delta F v s t u A 1 / 7$, $\Delta F v s t u A 2 / 8, \Delta F v s t u A 2 / 11, \Delta F v s t u A$ 2/12). Southern blot analysis of the mutants confirmed deletion of FvSTUA (Supplementary Fig. S1A). The absence of the 1,767-bp FvSTUA open reading frame (ORF) (Supplementary Fig. S1B) and the presence of the hygromycin-resistance gene (hyg) in the correct position of the genome, indicating homologous recombination and gene deletion (Supplementary Fig. S1C), were confirmed by PCR.

Mutant complementation with the WT FvStuA gene was unsuccessful after several attempts. We believe this was due to a combination of reasons, including a very low rate of protoplast production compared with WT, likely due to cell-wall alterations in the mutants, a low rate of transformation, and a very low rate of cotransformation. Cotransformants carrying the expected complementing fragments were not phenotypically complemented. To compensate for the lack of molecular complementation, we relied upon cosegregating progeny to confirm the phenotypic effect of the mutations. Three crosses of $\Delta F v$ stuA mutants $1 / 2,1 / 7$, and $2 / 8$ with $F$. verticillioides A00999 were similarly fertile in terms of the number of perithecia and the number of ascospores produced and were comparable to a cross between the WT M3125 and A00999 strains. Random ascospore progeny of the crosses were examined for hyg resistance, which would indicate inheritance of the $\Delta F v s t u A$ mutation. Twenty-four progeny from each cross were scored for their resistance to hyg on potato dextrose agar (PDA) and PDAhyg 150 plates. The progeny did not segregate in a 1:1 manner consistent with that expected for a single gene mutation and only three or four of the progeny from each cross were hyg-resistant. This result supports a likely strong fitness cost to the $\Delta F v s t u A$ mutation.

\section{$\Delta F v s t u A$ mutants display reduced macro and microconidiation. \\ To examine the role of $F v S T U A$ in the macroconidiation of $F$. verticillioides, M3125 and the $\Delta F v$ stuA mutants were grown on carboxy methyl cellulose agar (CMC) agar (which is stim- ulatory to macroconidial production) for 10 days, and we}


qualitatively observed the production of macroconidia. The WT strain produced abundant, characteristic, canoe-shaped macroconidia (Fig. 3A). In contrast, there was little to no production of macroconidia in the $\triangle F v s t u A$ mutants (Fig. 3B), except for
$\Delta$ FvstuAl/7 (Fig. 3C), which produced abundant macroconidia like the WT. Microconidiation was significantly reduced in all the $\Delta F v s t u A$ mutants; the mutants exhibited a 10 - to 100 -fold decrease in conidial production compared with WT (Table 1).

\section{A}

Fusarium verticillioides StuA

Neurospora crassa

Asm1

\section{Saccharomyces cerevisiae} Phd1
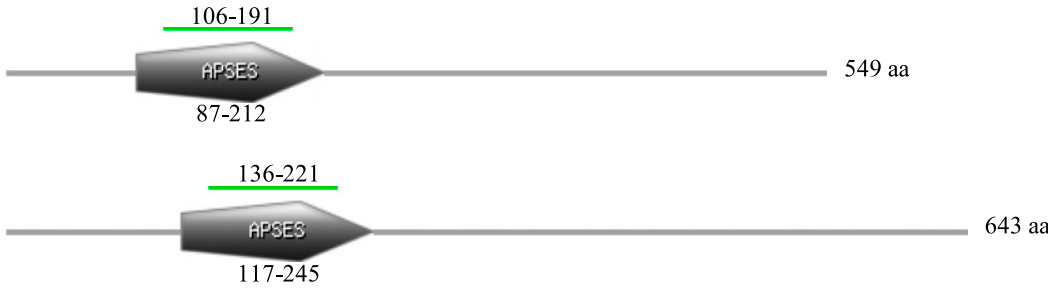

208-253

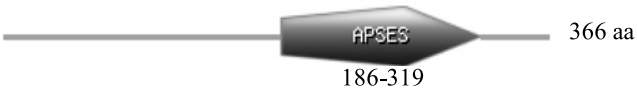

Saccharomyces cerevisiae Sok2

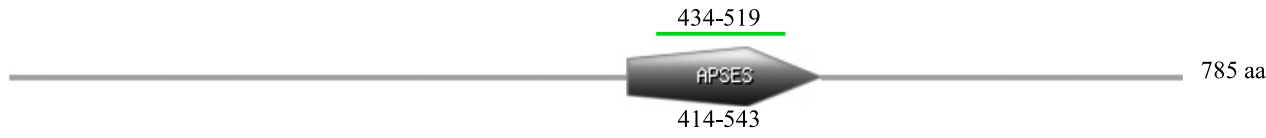

\section{Candida albicans \\ Efg1}

Aspergillus nidulans

StuA

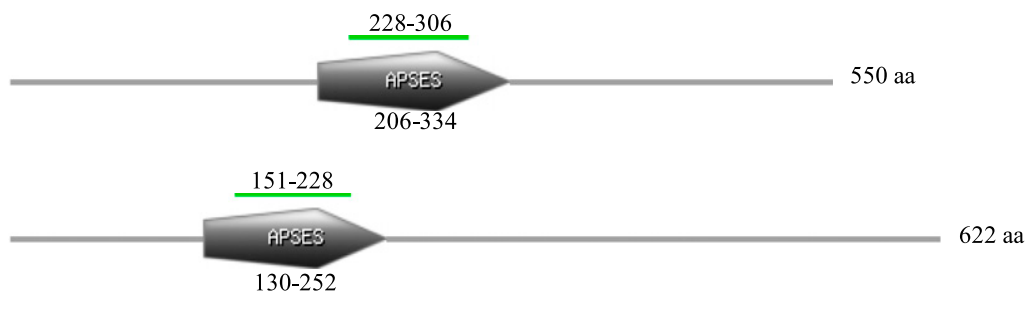

B

\section{Fusarium verticillioides StuA}

\section{Fusarium fujikuroi Asm1 relative}

\section{Fusarium graminearum} APSES transcription factor
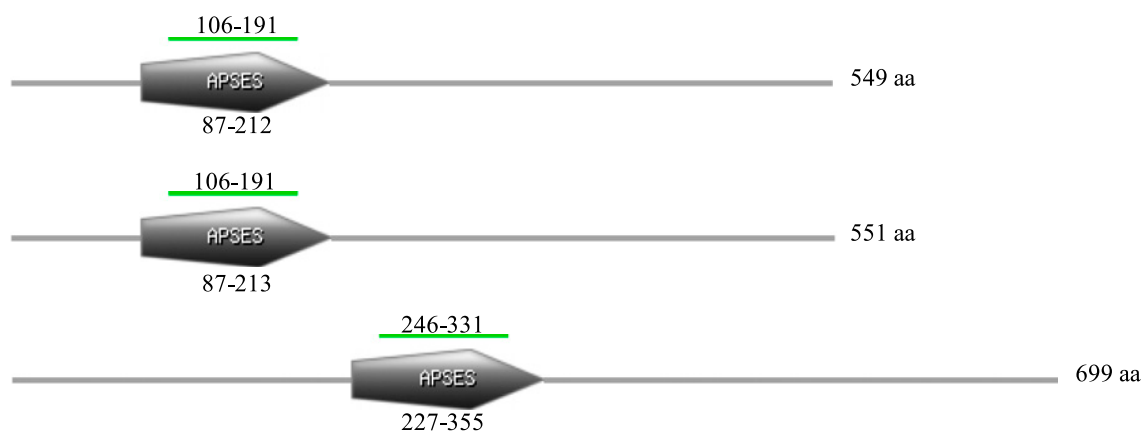

\section{Fusarium oxysporum StuA}

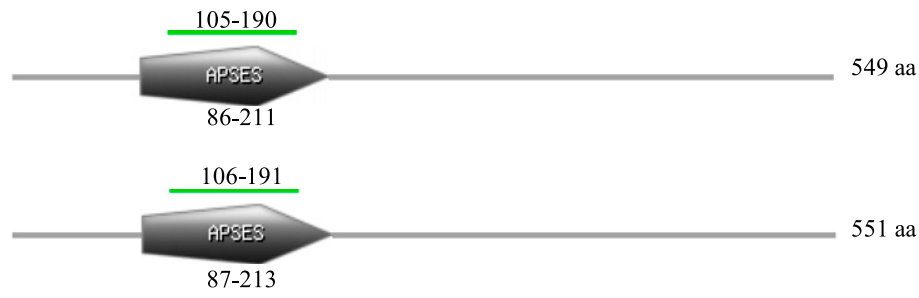

Fusarium proliferatum Asm1 relative

\section{aa}


Although growth and microconidiation results for all the deletion strains were similar, each mutant displayed slightly different characteristics. Rather than select one mutant strain for further analysis, three of the six deletion strains were selected for additional functional assays and transcriptomal characterization. $\Delta F v s t u A 1 / 2, \Delta F v s t u A 1 / 7$, and $\Delta F v s t u A 2 / 8$ were selected for further analysis, since these three displayed reduced mycelial growth and microconidiation compared with the other mutants and were thus considered to be more indicative of the original mutant phenotype prior to undergoing ameliorating second-site mutations.

\section{$\Delta F v s t u A$ mutants display stunted aerial hyphae, growth, and reduced hydrophobicity.}

The growth of the six deletion strains $(\Delta F v s t u A 1 / 2$, $\Delta F v s t u A 1 / 3, \Delta F v s t u A 1 / 7, \Delta F v s t u A 2 / 8, \Delta F v s t u A 2 / 11$, and $\Delta F v s t u A 2 / 12)$ was assessed on PDA. Colony diameter was measured after 5 and 10 days of growth. All mutants exhibited severely stunted aerial hyphae (Fig. 4A). A closer examination of mycelial growth revealed that the hyphae appeared to be embedded in the medium (not shown). Radial growth of the mutants was slightly but significantly reduced at the timepoints measured as compared with that of WT (M3125) on PDA (Fig. 4B). Although variation in pigmentation was observed in the mutant strains on plates and in liquid potato dextrose broth (PDB) compared with WT (Fig. 4A; Supplementary Fig. S2), it was not consistent across all the deletion strains. Additionally, sectoring was also commonly observed in some of the mutants (Supplementary Fig. S3).

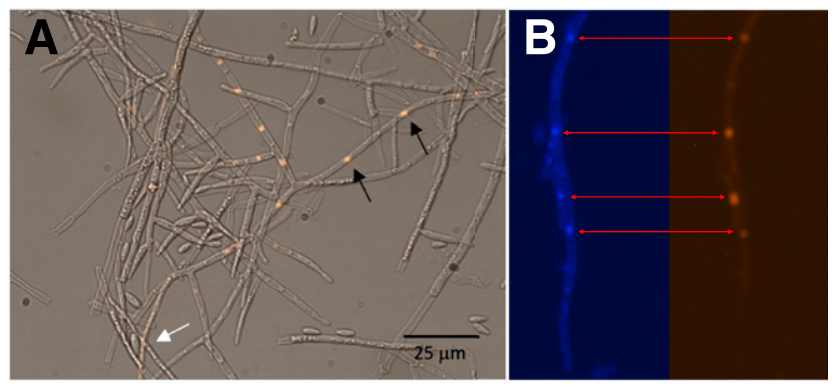

Fig. 2. Localization of FvSTUA-tdTomato in hyphae of Fusarium verticillioides. A, Black arrows indicate location of fluorescent nuclei, white arrow indicates diffuse cytoplasmic fluorescence. B, Colocalization (red arrows) of 4',6-diamidino-2-phenylindole nuclear and StuA-tdTomato fusion protein fluorescent signals.
The mycelial surface of $\Delta F v s t u A$-deletion strains also exhibited reduced hydrophobicity compared with the WT M3125. A 10- $\mu$ l water droplet placed on the surface of WT culture on PDA persisted for more than $2 \mathrm{~h}$. However, the droplet dispersed in less than $30 \mathrm{~s}$ on the surface of the $\Delta F v s t u A$-deletion strains (Fig. 5), demonstrating the "wettability" of the deletion strains. Together these results suggest that FvSTUA is involved in but is not indispensable for vegetative growth and asexual sporulation. It is also required for hydrophobicity. The reduced expression of one or more hydrophobin genes may be responsible, at least in part, for the reduction in aerial hyphae.

\section{$\Delta F v s t u A$ mutants display reduced fumonisin production.}

To investigate the impact of FvSTUA on fumonisin production, M3125, the mutants $\Delta F v s t u A 1 / 2, \Delta F v s t u A 1 / 7$, and $\Delta F v s t u A 2 / 8$, and two hyg-resistant and hyg-sensitive progeny of each mutant were grown in GYAM medium ( $0.24 \mathrm{M}$ glucose, $0.05 \%$ yeast extract, $8 \mathrm{mM}$ L-asparagine, $5 \mathrm{mM}$ malic acid, $1.7 \mathrm{mM} \mathrm{NaCl}, 4.4 \mathrm{mM} \mathrm{K}_{2} \mathrm{HPO}_{4}, 2 \mathrm{mM} \mathrm{MgSO}_{4}$, and $8.8 \mathrm{mM}$ $\mathrm{CaCl}_{2}, \mathrm{pH} 3.0$ ) for 7 days at $27^{\circ} \mathrm{C}$ in the dark. When normalized to fungal biomass, the levels of fumonisin produced by the mutants were extremely low, and hence, a $\log _{10}$-based transformation was conducted to more accurately interpret and represent the data graphically (Fig. 6). The mutants produced significantly lower fumonisin levels than the WT. In general, $\mathrm{FB}_{1}$ was the individual fumonisin produced in the largest quantities, followed by $\mathrm{FB}_{2}$ and $\mathrm{FB}_{3}$.

\section{$\Delta F v s t u A$ mutants display decreased virulence.}

To test the virulence of the $\Delta F v s t u A$ mutants, maize seedling assays were conducted on the cultivar Silver Queen. The disease

Table 1. Microconidiation of wild type (M3125) and $\Delta F v s t u A$ mutants of Fusarium verticillioides

\begin{tabular}{lcc}
\hline Strain & Mean conidia at 5 dpia & Mean conidia 10 dpi \\
\hline M3125 & $3.090 \times 10^{8}$ & $5.873 \times 10^{8}$ \\
$\Delta$ FvstuA1/2 & $2.485 \times 10^{6}$ & $4.445 \times 10^{6}$ \\
$\Delta$ FvstuA1/3 & $6.215 \times 10^{6}$ & $1.176 \times 10^{7}$ \\
$\Delta$ FvstuA1/7 & $1.020 \times 10^{7}$ & $5.640 \times 10^{7}$ \\
$\Delta$ FvstuA2/8 & $1.436 \times 10^{7}$ & $4.840 \times 10^{7}$ \\
$\Delta$ FvstuA2/11 & $7.085 \times 10^{6}$ & $1.065 \times 10^{7}$ \\
$\Delta$ FvstuA2/12 & $5.026 \times 10^{7}$ & $6.893 \times 10^{6}$ \\
\hline
\end{tabular}

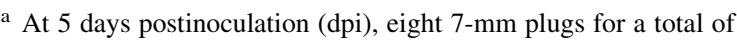
approximately $308 \mathrm{~mm}^{2}$. At both 5 and $10 \mathrm{dpi}$, the mean is from three independent repeats with three replicates for each strain.

b At $10 \mathrm{dpi}$, twelve 7-mm plugs for a total of approximately $462 \mathrm{~mm}^{2}$.
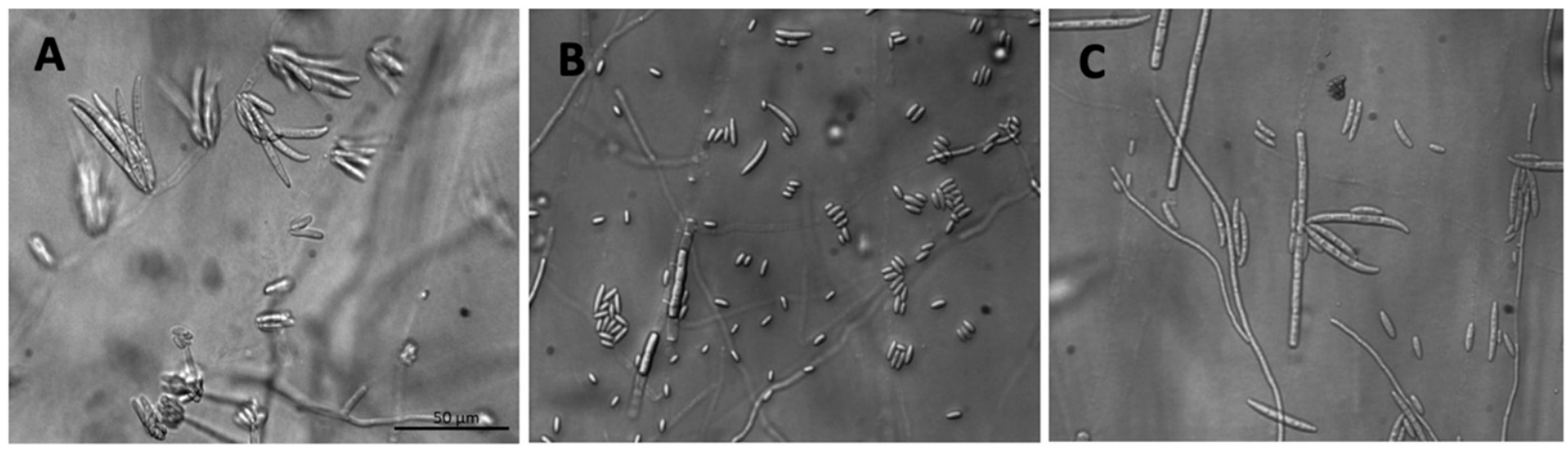

Fig. 3. Macroconidiation of Fusarium verticillioides wild type (M3125) and $\Delta F v s t u A$ deletion mutants grown at $27^{\circ} \mathrm{C}$ for 15 days on carboxy methyl cellulose (CMC) agar medium, which induces macroconidiation for Fusarium spp. A, Characteristic septate and canoe-shaped macroconidia seen for M3125 on CMC agar. B, Representative picture of $\Delta F v s t u A$ deletion strains showing only microconidia on CMC agar, no macroconidia. $\Delta F v s t u A 2 / 8$ shown here. C, $\Delta F v s t u A 1 / 7$ deletion mutant showing production of macroconidia on CMC agar. 
symptoms of the inoculated plants were measured as a decrease in shoot height and fresh shoot weight with respect to that of uninoculated control seedlings 14 days after planting (dap). The average height and weight of the shoots in response to the different treatments are shown in Figure 7B and C, respectively.
Analysis of variance (ANOVA) followed by comparison of least square means showed that there were significant differences between the heights and weights of shoots of the control seedlings and those inoculated with WT. The height of the seedlings inoculated with $\Delta F v s t u A 1 / 2$ and $\Delta F v s t u A 1 / 7$ differed significantly

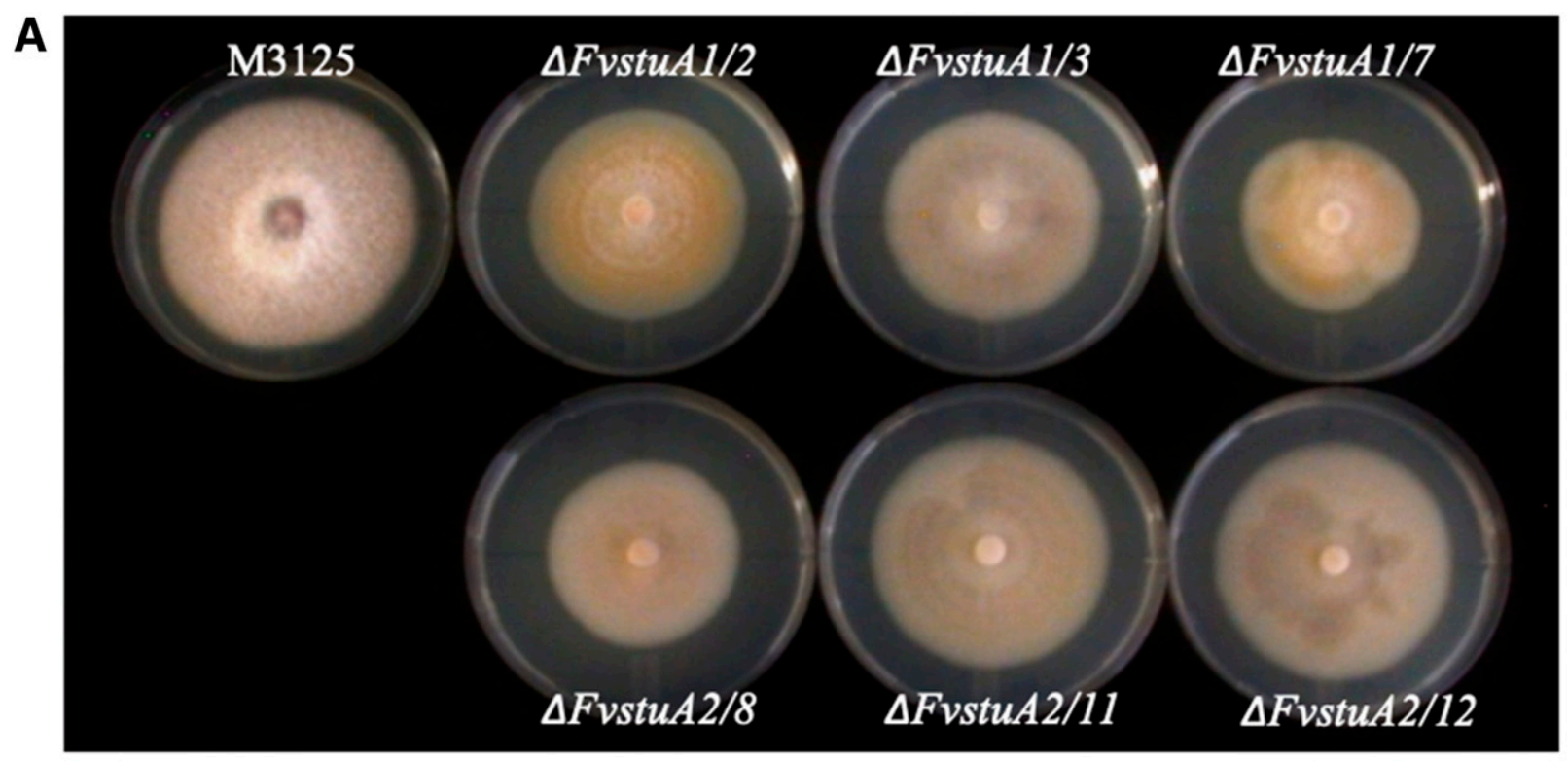

B

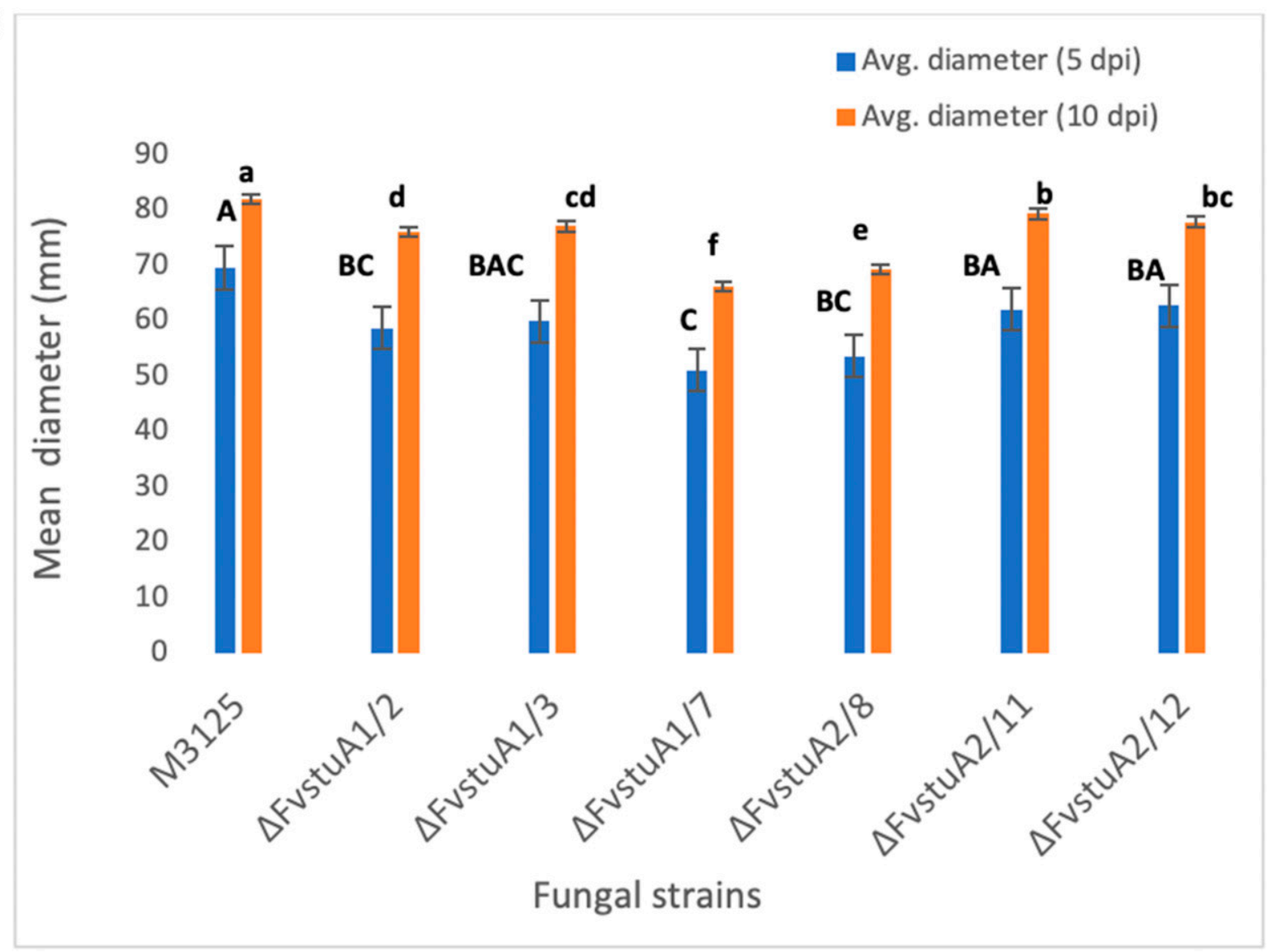

Fig. 4. Morphology and mycelial growth of Fusarium verticillioides wild type (M3125) and $\Delta F v$ stuA deletion mutants on potato dextrose agar (PDA), grown at $27^{\circ} \mathrm{C}$ for 7 days. A, The M3125 strain shows an abundance of white, fluffy aerial hyphae, whereas all the $\Delta F v s t u A$ mutants exhibit stunted aerial hyphae. B, Diameter of radial growth of the $\Delta F v s t u A$ mutants compared with M3125 on PDA, grown from $7-\mathrm{mm}$ transferred mycelial plugs at $27^{\circ} \mathrm{C}$ in the dark for 5 and 10 days postinoculation (dpi). The assay was repeated three times with three replicates for each strain. Statistical analysis was done with analysis of variance followed by comparison of least square means. Diameters of strains with different letters are significantly different at $P \leq 0.05$. 
from the heights of the WT-inoculated seedlings (Fig. 7B). Additionally, they were not significantly different from the height of the control seedlings and did not show stunted growth (Fig. 7A and 7B). With regards to fresh weight, although both $\Delta F v s t u A 1 / 2$ and $\Delta F v s t u A 1 / 7$ had greater fresh weight than the WT treatment $(P \leq 0.05)$, only $\Delta F v s t u A 1 / 2$ treatment was significantly higher than WT treatment. Interestingly, heights and weights of $\Delta F v s t u A 2 / 8$-inoculated seedlings differed significantly from those of the other two mutant-inoculated seedlings but were very similar to those of the WT-inoculated seedlings, indicating no difference in virulence between $\Delta F v s t u A 2 / 8$ and the WT strain (Fig. 7).

\section{FvSTUA regulates sporulation, pathogenicity, and toxin production-associated genes.}

To investigate the molecular mechanisms by which FvSTUA affects sporulation and virulence in $F$. verticillioides, transcriptomic analyses of WT M3125 and the mutants $\Delta F v$ StuAl/2, $\Delta F v S t u A 1 / 7$, and $\Delta F v S t u A 2 / 8$ were conducted in two types of media, i.e., PDB, a medium used commonly for growing fungal cultures, and GYAM medium, which is commonly used as conducive to fumonisin production.

Conidiation/sporulation-associated genes. Putative homologs of fluG, fluD, and fluC (ADAl) were differentially regulated in FvstuA mutants. FLUG in $\triangle F v s t u A$ deletion strains was expressed approximately 2.7 - to 10.5 -fold lower in the mutant strains compared with WT (Table 2). In contrast, FLUD and ADAl were slightly upregulated. However, the expression of several other genes involved in conidiation in $F$. verticillioides (FLBE, FLBB, WETA, MEDA, ABAA, FCC1) did not differ significantly in the $\triangle F v$ stuA mutants compared with the WT sample. Interestingly, expression of a gene annotated as $\mathrm{N}$-acetyl polyamine oxidase (FVEG_12823) was significantly downregulated in all three mutant strains, with an expression level 30- to 294-fold lower than WT (Table 2). The enzyme putatively encoded by this gene is part of the polyamine retroconversion pathway in fungi, responsible for the conversion of $\mathrm{N}$-acetylspermine to putrescine, and polyamines including putrescine have been found to be required for cell proliferation and differentiation in fungi (Valdés-Santiago et al. 2012). Three putative catalase genes (FVEG_03348, catalase-1 FVEG_05591, and catalase-2 FVEG_12611) were differentially expressed in the mutants; FVEG_03348 was significantly downregulated and catalase-2 was upregulated. In contrast, two catalase-peroxidase genes (FVEG_12888 and FVEG_10866) were upregulated in their expression. The putative catalase-1 (FVEG_05591), similar to spore-specific catalases in Neurospora crassa and Gibberella zeae (Niu et al. 2015), was significantly downregulated in two of the mutants but slightly upregulated in one $(\Delta F v s t u A 1 / 2)$ mutant (Table 2). Surprisingly, the expression of FVEG_12826, similar to $F L$ in Neurospora crassa, which is critical for conidial bud morphogenesis and production (Bailey and Ebbole 1998), was upregulated twofold across the mutants.

Hydrophobins have been shown to influence conidiation in F. verticillioides (Fuchs et al. 2004; Ridenour and Bluhm 2014). Examination of hydrophobin gene expression revealed that each $\Delta F v s t u A$ mutant had at least four of the eight $H Y D$ genes differentially regulated. However, except for $H Y D 8$, the other genes were not consistently differentially expressed across all the mutants. HYD8 (FVEG_10008) was significantly downregulated in both $\Delta F v s t u A 1 / 7$ and $\Delta F v s t u A 2 / 8$ by six- and 21 -fold, respectively; although necessarily high, exact fold change could not be calculated in $\Delta F v s t u A 1 / 2$ because of a null expression value (Supplementary Table S1). Since each mutant showed increased colony wetting and had a number of $H Y D$ genes downregulated, the transcription factor FvSTUA clearly plays a role in regulating hydrophobins. Furthermore, the differential regulation of these genes might be affecting conidiation and aerial hypha formation, which were both reduced in the $\Delta F v s t u A$ mutants.

Pathogenicity- and virulence-associated genes. A search of F. verticillioides pathogenicity-associated genes listed in PHIbase (Urban et al. 2017) for possible genes affected by FvSTUA revealed FvHAP3, Mads1, FvSO, and UBL1 (FVEG_08087, FVEG_01965, FVEG_08055, and FVEG_01321, respectively) to be differentially expressed across the three mutant strains. All the genes except for FvSO (FVEG_08055) were slightly downregulated by at least 1.5 -fold. FvSO, on the other hand, was upregulated by roughly 1.5 -fold in the mutants (Table 2).
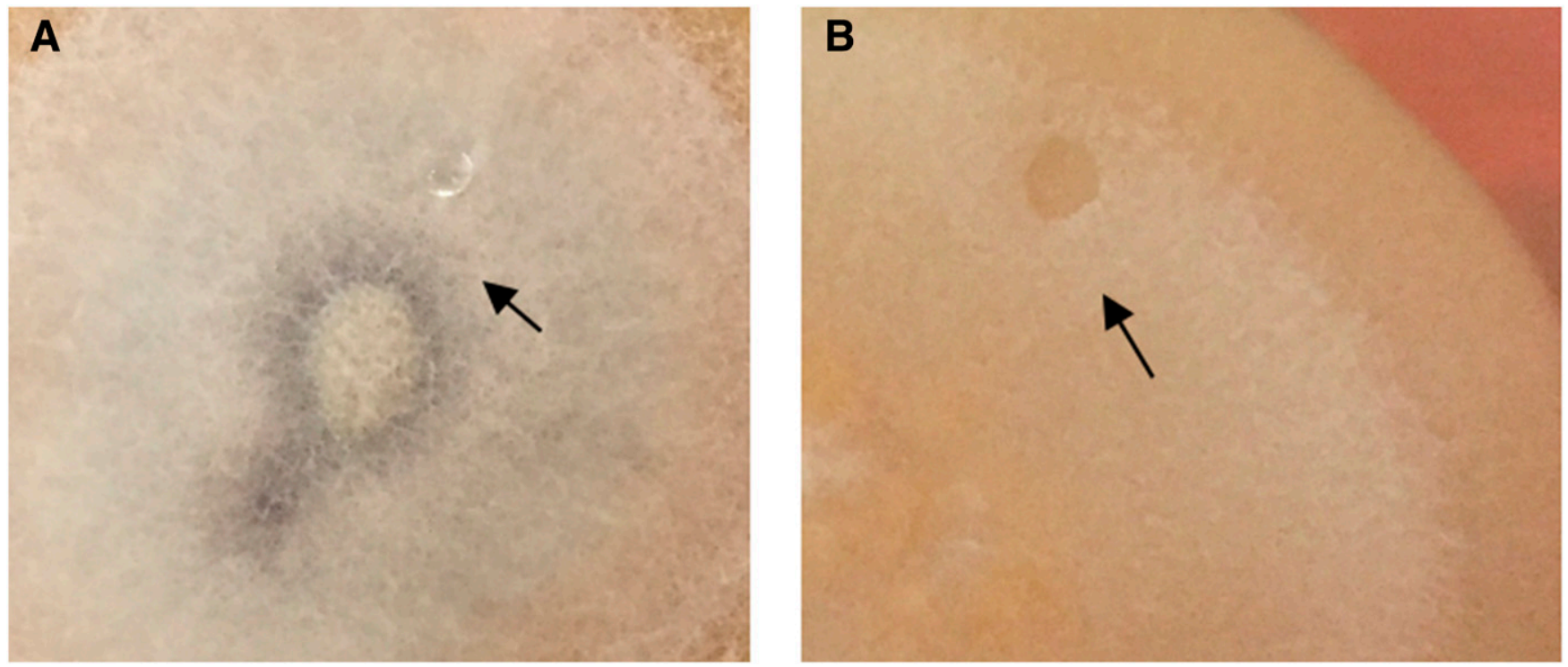

Fig. 5. Hydrophobicity test of Fusarium verticillioides wild-type (M3125) and $\Delta F v s t u A$ deletion mutants grown on potato dextrose agar (PDA) for 5 days, testing for persistence of water droplets on mycelial growth. A, Hydrophobicity of a 10- $\mu$ l water droplet on M3125 mycelial growth on PDA, droplet was persistent $>2 \mathrm{~h}$. B, Hydrophobicity of a $10-\mu \mathrm{l}$ water droplet on a $\Delta F v s t u A$ mutant, droplet dispersed in $<30 \mathrm{~s}$. The dispersion of water droplets shown here is representative of all the $\Delta F v s t u A$ mutants. Arrows point to the water droplets. 
HAP3, part of the HAP complex, and Mads1 are transcription factors (Ortiz and Shim 2013; Ridenour et al. 2014). FvSO and $U B L 1$ encode putative WW domain-containing signaling proteins required for anastomosis and other growth and developmental phenotypes and an E3 ubiquitin ligase, respectively (Ridenour et al. 2014; Guo et al. 2015). Interestingly, all these genes (except for Mads1) are also implicated in reduced or aberrant macro- or microconidiation, or both, and impaired vegetative growth. Madsl, although not involved in asexual sporulation in $F$. verticillioides, regulates sexual reproduction in the pathogen (Ortiz and Shim 2013). Together, these results indicate that FvSTUA impacts the expression of conidiation and pathogenicityassociated genes in $F$. verticillioides.

Fumonisin, fusarin $C$ and fusaric acid biosynthetic gene expression. To identify genes regulated by FvSTUA in toxin production, transcriptomic data were compared between WT M3125 and the three independent $\Delta F v s t u A$ mutants grown in GYAM, a medium conducive to toxin production. The RNA-Seq data indicated that the FUM and FUS genes were among the most differentially expressed in the $\Delta F v s t u A$ deletion strains (Table 3 ).

Expression of five genes (FUM6, FUM13, FUM14, FUM18, and $F U M 19$ ) from the FUM cluster was significantly downregulated
( $\geq 3$ fold), with expression of FUM6 being 18- to 39-fold lower than that of WT (Table 3). In contrast to the biosynthetic FUM genes, two of the genes flanking the FUM cluster, FVEG_00311 and FVEG_00312, were slightly upregulated across the mutants (Supplementary Table S2). Some of the other genes associated with the FUM cluster also showed differential regulation, but differential regulation was not observed across all the mutants (Supplementary Table S2).

Deleting the FvSTUA gene also had a significant effect on the expression of the mycotoxin fusarin $\mathrm{C}$ biosythentic cluster genes. The expression of the FUS genes (Brown et al. 2012) was at least 15 -fold lower in the mutant strains, with most of the genes expressing at a level 27- to 80-fold lower than that in WT. All the FUS genes, except FUS3, which showed differential expression only in $\Delta F v s t u A 1 / 2$ and $\Delta F v s t u A 1 / 7$, were significantly downregulated across the three mutants. A few of the genes had fold changes 200 to 700 times lower than WT (Table 3). An interesting observation was that the $F U B$ gene cluster involved in fusaric acid biosynthesis contained some of the most differentially expressed genes in the $\Delta F v s t u A l / 7$ deletion strain; seven of the 12 genes were downregulated, including two C6 transcription factors. In contrast, most of the differentially expressed

4

- Log10 FB1 means

- Log10 Total fumonisin means

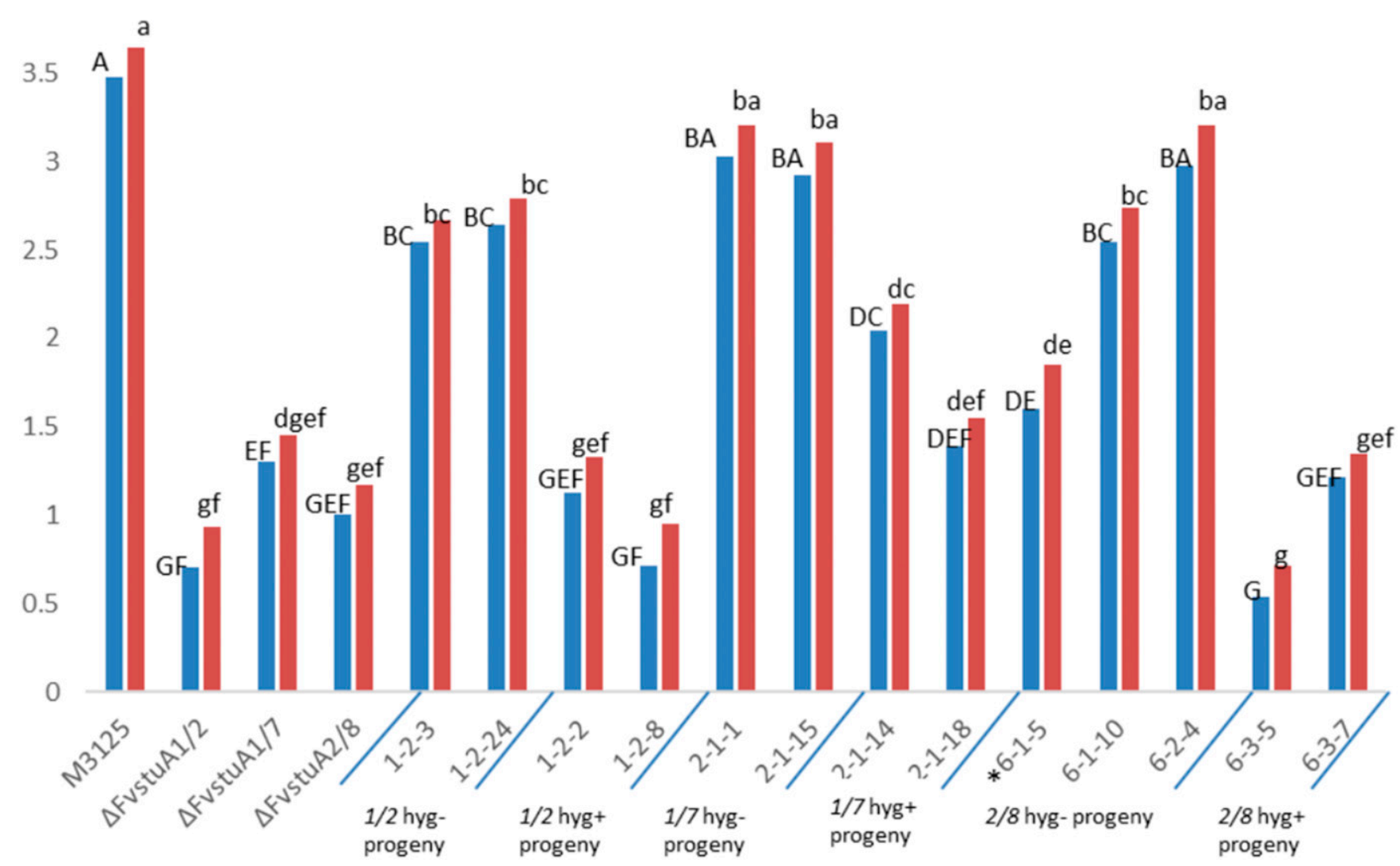

Fig. 6. Fumonisin production by Fusarium verticillioides wild type (M3125), $\Delta F v s t u A$ deletion strains, and progeny of $\Delta F v s t u A$ strains grown for 7 days in toxin-inducing GYAM medium at $27^{\circ} \mathrm{C}$ and $225 \mathrm{rpm}$ in an incubator-shaker. The bar graph shows $\log _{10}$ of $^{\mathrm{FB}} \mathrm{F}_{1}$ and $\log _{10}$ of total fumonisin levels of $\mathrm{M}_{3} 125$, $\Delta F v s t u A$ mutants, and their hyromycin (hyg)-sensitive and hyg-resistant progeny. The hyg-sensitive (hyg-) progeny did not inherit $\Delta F v s t u A$ mutation, since hyg marker is selection for $\Delta F v s t u A$ deletion, whereas the hyg-resistant (hyg+) progeny inherited the $\Delta F v s t u A$ mutation. Two hyg- and two hyg+ progeny of each $\Delta F v s t u A$ mutant were used in the assay to determine the heritability of the phenotypic effects of $\Delta F v s t u A$ mutation. The relatively high fumonisin production by the hyg- and low fumonisin production by hyg+ progeny indicate that the phenotypic effects of the $\Delta F v s t u A$ mutation are heritable. The experiment was conducted in triplicate with each treatment having three replicates. Analysis of variance followed by comparison of least square means was used for statistical analysis. Treatments that do not share a letter are significantly different at $P<0.05$. *6-1-5 is a hyg- progeny of $\Delta F v$ stuA2/8 but, contrary to other hyg- strains, showed low levels of fumonisin production, so another hyg-sensitive progeny 6-2-4 was used in the last two repeats in addition to 6-1-5. 
genes in the FUB (Brown et al. 2015) cluster in $\Delta F v s t u A 1 / 2$ and $\Delta F v s t u A 2 / 8$ were slightly upregulated (Table 3 ).

\section{DISCUSSION}

In fungi, the APSES transcription factors are known to regulate fungal morphogenesis and development in some broadly shared ways but also in species-unique patterns. These transcription factors also participate in controlling virulence traits (Ramírez-Zavala and Domínguez 2008; Zhao et al. 2015). The FvSTUA protein sequence showed the highest homology with FoSTUA of Fusarium oxysporum f. sp. cubense race 4 (98\%). FvSTUA, like other APSES proteins, has a conserved APSES domain and an additional KilA N-terminal domain that is found

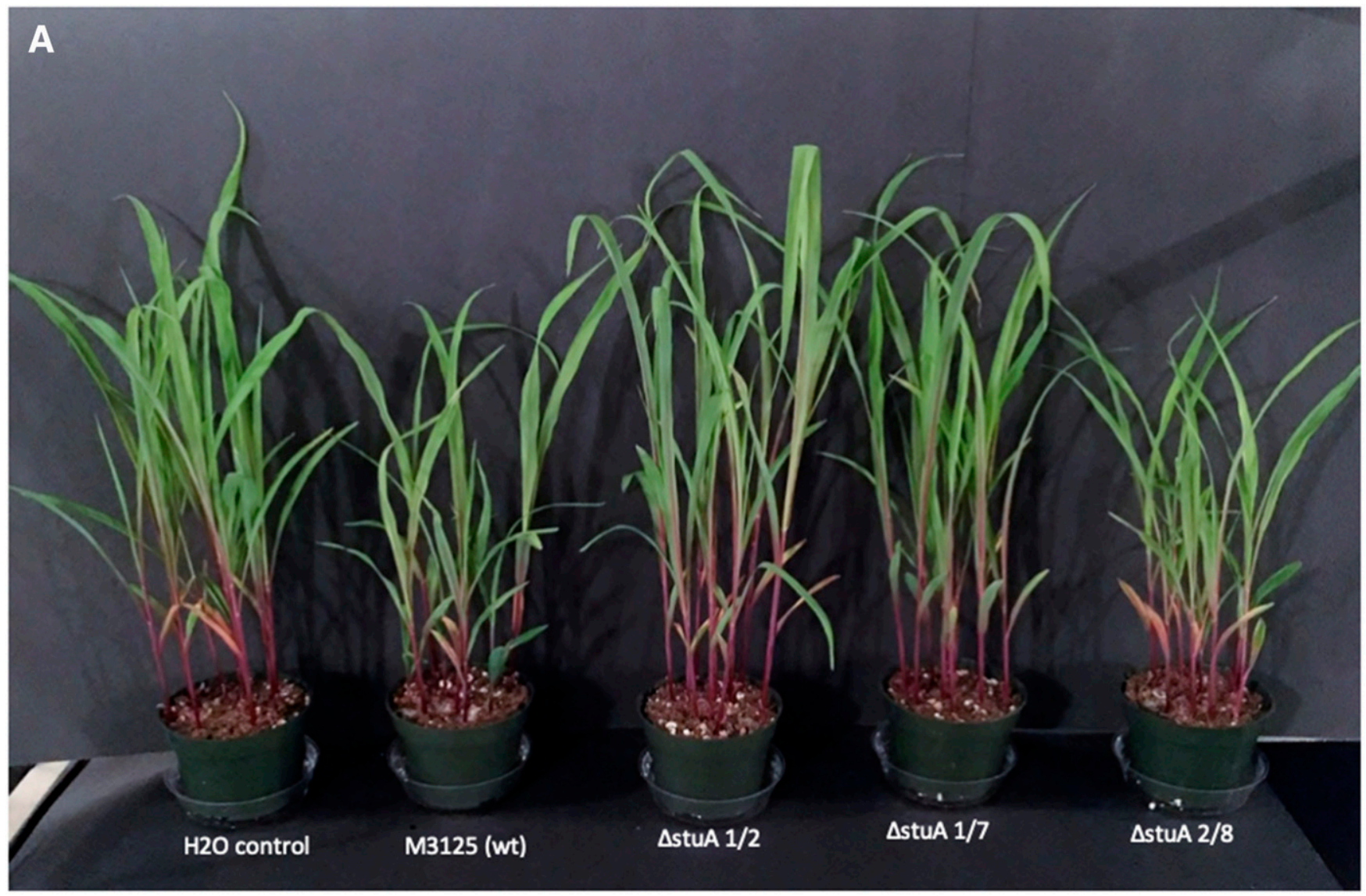

B

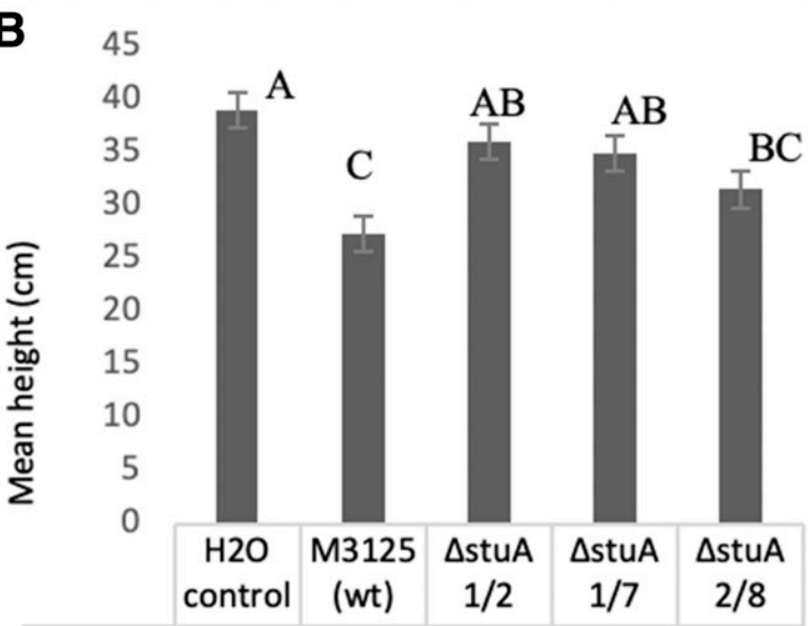

Height 38.907827.244235.957834.796631.4552

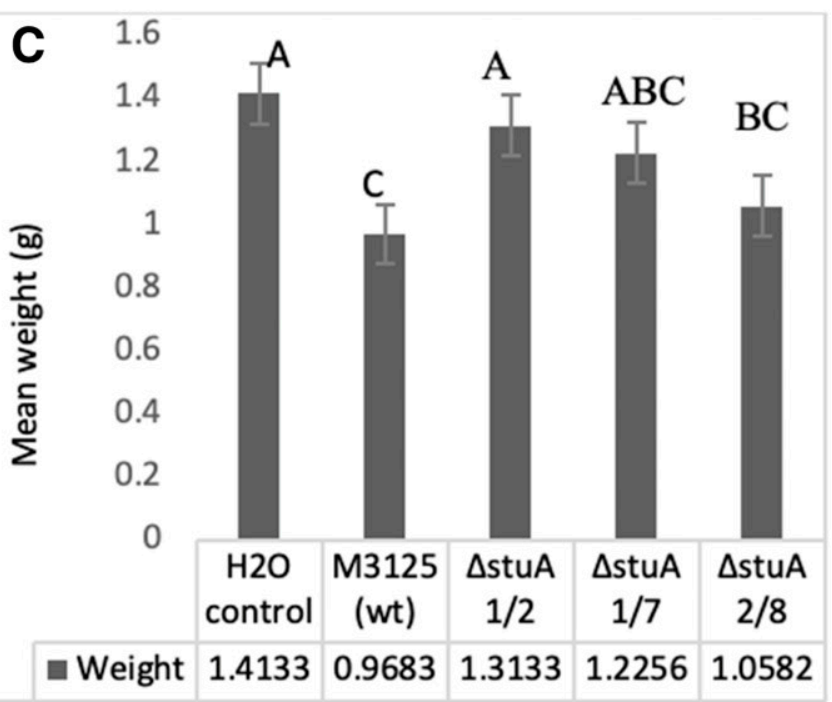

Fig. 7. Virulence of Fusarium verticillioides wild type (M3125) and $\Delta F v$ stuA deletion mutants on 14-day-old maize (cultivar Silver Queen) seedlings. A, Silver Queen seedlings (14 days after planting) grown from seed inoculated with water control, M3125 (wt), $\Delta F v s t u A 1 / 2, \Delta F v s t u A 1 / 7$, and $\Delta F v s t u A 2 / 8$ (from left to right). Seedlings inoculated with M3125 show the characteristic growth stunting. However, growth of seedlings inoculated with $\Delta F v s t u A 1 / 2$ and $\Delta F v s t u A 1 / 7$ are similar to those of the water control. B, Mean height (in centimeters) of shoots from seedlings inoculated with water (control), WT (M3125), and the $\Delta F v s t u A$ mutants. C, Mean fresh weight (in grams) of shoots from seedlings inoculated with water (control), WT (M3125), and the $\Delta F v s t u A$ mutants. The experiment was conducted in triplicate with each treatment having three replicates of 10 seedlings. Error bars represent standard deviation. Statistical analysis was done with analysis of variance followed by comparison of least square means. Mean height (and mean weight) of treatments that do not share a letter are significantly different at $P \leq 0.05$. 
exclusively in StuA proteins (Zhao et al. 2015). The localization of FvSTUA to the nucleus, as demonstrated here, provides support consistent with its role as a transcription factor (Fig. $2)$. To investigate the role of this transcription factor in $F$. verticillioides, a gene-deletion approach was undertaken. In-vitro observations of reduced growth and stunted aerial hyphae of the $\Delta F v s t u A$ deletion mutants (Fig. 4) is consistent with that of the stuA mutants of Glomerella cingulata, F. oxysporum, Neurospora crassa, and A. nidulans (Aramayo et al. 1996; Clutterbuck 1969; Ohara and Tsuge 2004; Tong et al. 2007). Hydrophobins are known to play a role in the formation of aerial hyphae (Kershaw and Talbot 1998; Teertstra et al. 2006), and several stuA mutants have demonstrated increased wettability (Lysøe et al. 2011). The reduced hydrophobicity of the $\Delta F v s t u A$ deletion strains, together with the downregulation of the $H Y D$ genes, particularly $H Y D 8$, could be contributing to their phenotypic differences compared with WT samples (Supplementary Table S1).

Since the deletion of $s t u A$ in most fungi studied led to a reduction in sporulation (Zhao et al. 2015), conidiation was investigated in the $\Delta F v s t u A$ deletion strains. The significantly reduced microconidiation (10 to 100 -fold lower than WT)

Table 2. Differential expression of sporulation and pathogenicity-associated genes in $\Delta F v$ stuA mutants of Fusarium verticillioides ${ }^{\mathrm{a}}$

\begin{tabular}{|c|c|c|c|c|}
\hline Gene name & Gene ID & $\begin{array}{c}\log _{2} \text { (fold change) } \\
\Delta F v \sin A 1 / 2\end{array}$ & $\begin{array}{c}\log _{2} \text { (fold change) } \\
\Delta F v s t u A 1 / 7\end{array}$ & $\begin{array}{c}\log _{2} \text { (fold change) } \\
\Delta F v \text { stu } A 2 / 8\end{array}$ \\
\hline flu $G$ homolog & FVEG_02766 & -1.78416 & -3.43898 & -1.48506 \\
\hline flbD homolog & FVEG_07339 & 1.32424 & 0.942554 & 1.90263 \\
\hline$A D A 1 / f l b C$ homolog & FVEG_08125 & 1.64024 & 1.25573 & 0.873856 \\
\hline $\mathrm{N}$-acetyl polyamine oxidase & FVEG_12823 & -8.20167 & -4.97458 & -5.7991 \\
\hline Catalase & FVEG_03348 & -2.89476 & -2.50942 & -2.50884 \\
\hline Catalase-peroxidase 2 & FVEG_12888 & 3.17677 & 3.19454 & 2.17947 \\
\hline Catalase-peroxidase 2 & FVEG_10866 & 0.436005 & 1.88042 & 0.68838 \\
\hline Catalase-1 & FVEG_05591 & 0.682191 & -1.08844 & -0.841281 \\
\hline Catalase-2 & FVEG_12611 & 0.446757 & 1.58277 & 0.538313 \\
\hline $\begin{array}{l}\text { FLF1 (similar to } f l \text { in Neurospora } \\
\text { crassa) }\end{array}$ & FVEG_12826 & 1.01493 & 1.05247 & 1.07336 \\
\hline HAP3 & FVEG_08087 & -0.545496 & -0.556449 & -0.559553 \\
\hline Mads1 & FVEG_01965 & -0.696916 & -0.493194 & -0.502759 \\
\hline$F v S O$ & FVEG_08055 & 0.669465 & 1.09325 & 0.748711 \\
\hline
\end{tabular}

${ }^{\mathrm{a}}$ RNA-Seq analysis was performed on wild-type M3125 and three independent deletion mutants $(\Delta F v s t u A 1 / 2, \Delta F v s t u A 1 / 7$, and $\Delta F v s t u A 2 / 8)$ were grown in potato dextrose broth at $27^{\circ} \mathrm{C}$ at $225 \mathrm{rpm}$ in the dark for $30 \mathrm{~h}$ with the caps unsnapped. Data analysis was done with the Cufflinks suite of tools available on the Galaxy server. All expression values listed are significantly differentially regulated at $P<0.05$.

Table 3. Expression of genes from fumonisin, fusarin $\mathrm{C}$ and fusaric acid biosynthesis clusters in Fusarium verticillioides ${ }^{\mathrm{a}}$

\begin{tabular}{|c|c|c|c|c|}
\hline \multirow[b]{2}{*}{ Gene name } & \multirow[b]{2}{*}{ Gene ID } & \multicolumn{3}{|c|}{$\log _{2}$ (fold change) } \\
\hline & & $\Delta F v s t u A 1 / 2^{\mathrm{b}}$ & $\Delta F v s t u A 1 / 7$ & $\Delta F v s t u A 2 / 8$ \\
\hline \multicolumn{5}{|c|}{ Fumonisin cluster } \\
\hline FUM6 & FVEG_00317 & -4.19146 & -5.32154 & -4.46542 \\
\hline FUM13 & FVEG_00324 & -1.922 & -2.76865 & -3.39685 \\
\hline FUM14 & FVEG_00325 & -3.84952 & -4.75784 & -4.24913 \\
\hline FUM18 & FVEG_00328 & -2.48756 & -3.93021 & -4.73382 \\
\hline FUM19 & FVEG_00329 & -2.11842 & -3.39845 & -3.20356 \\
\hline \multicolumn{5}{|c|}{ Fusarin cluster } \\
\hline FUS9 & FVEG_11078 & -5.27435 & -6.80385 & -6.37859 \\
\hline FUS8 & FVEG_11079 & -5.53856 & -8.51503 & -8.47858 \\
\hline FUS7 & FVEG_11080 & -5.57083 & -8.68598 & -8.31649 \\
\hline FUS6 & FVEG_11081 & -3.98021 & -7.47052 & -6.19161 \\
\hline FUS5 & FVEG_11082 & -5.29485 & -8.64238 & -9.68542 \\
\hline FUS4 & FVEG_11083 & -4.88123 & -7.65443 & -7.83345 \\
\hline FUS3 & FVEG_11084 & -4.81477 & -8.194 & $\mathrm{NS}^{\mathrm{c}}$ \\
\hline FUS2 & FVEG_11085 & -4.71549 & -8.03958 & -9.10511 \\
\hline FUS1 & FVEG_11086 & -4.86098 & -8.53382 & -9.65758 \\
\hline \multicolumn{5}{|c|}{ Fusaric acid cluster } \\
\hline FUB12 & FVEG_12534 & NS & -2.35978 & NS \\
\hline FUB11 & FVEG_12533 & NS & -3.91248 & -0.510407 \\
\hline FUB10 & FVEG_12532 & NS & -3.79435 & -0.407778 \\
\hline$F U B 9$ & FVEG_12531 & NS & -6.82616 & NS \\
\hline$F U B 8$ & FVEG_12530 & 0.868025 & -6.94499 & 0.856905 \\
\hline FUB7 & FVEG_12529 & NS & -6.52126 & NS \\
\hline FUB6 & FVEG_12528 & 1.14598 & -6.2238 & 1.39859 \\
\hline Aldolase & FVEG_12527 & 1.76597 & -5.59801 & 1.64923 \\
\hline$F U B 2$ & FVEG_12522 & 1.17182 & -5.57864 & 1.29643 \\
\hline FUB3 & FVEG_12521 & 1.09005 & -6.16427 & 0.973627 \\
\hline$F U B 4$ & FVEG_12520 & 0.619585 & -6.84012 & 1.39855 \\
\hline FUB5 & FVEG_12519 & 0.9724 & -6.46132 & 1.35715 \\
\hline
\end{tabular}


(Table 1) and near-elimination of macroconidiation (Fig. 3) of the $\triangle F v$ stuA deletion strains implies the importance of FvSTUA in sporulation of $F$. verticillioides. The reduced microconidiation is also consistent with a previous study by other authors of a FvSTUA gene-deletion mutant (Malapi-Wight et al. 2014).

Mining of RNA-Seq data identified a number of genes associated with conidiation that could be regulated by FvSTUA. Of six F. verticillioides homologs comprising the conidiation pathway in A. nidulans (Malapi-Wight et al. 2014), only three ( $F L U G, F L B D, F L B C$ ) were differentially expressed across all three $\Delta F v s t u A$-deletion strains in this study (Table 2). Based on a model of sporulation genetic circuitry in A. nidulans, these three genes are upstream of FvSTUA, which might explain the upregulation of $A D A 1$ and $F L B D$ in the deletion strains. Another group of genes associated with sporulation are spore-specific catalases (Giles et al. 2006). Mutants of one or both CATA and CATB in Magnaportha grise $a$ and $A$. nidulans exhibited reduced conidiation in addition to increased sensitivity to hydrogen peroxide (Navarro et al. 1996; Skamnioti et al. 2007). The inconsistent differential expression of a putative spore-specific catalase-1 (FVEG_05591) (Table 2) across the different mutants made it difficult to determine the negative or positive regulation of the gene by FvSTUA. However, other catalases and catalaseperoxidase-encoding genes (Table 2) appear to be upregulated, which is inconsistent with reports of reduced catalase production in the stuA mutants of A. nidulans, A. fumigatus, and F. graminearum (Scherer et al. 2002; Shephard et al. 1996; Lysøe et al. 2011). Interestingly, in $F$. graminearum, the expression of two catalase genes in the FgstuA mutant that exhibited increased expression in culture medium decreased during wheat infection. This situation might also be the case for $F$. verticillioides. Supporting this hypothesis is the fact that the catalase-peroxidases FVEG_12888 and FVEG_10866 appear to protect $F$. verticillioides from exogenous oxidative stress, which may occur during plant infection (Gao et. al 2018).

An intriguing preliminary discovery from the expression data were the significant downregulation of a gene encoding $\mathrm{N}$ acetyl polyamine oxidase that catalyzes a step in the retroconversion pathway of spermine to putrescine in fungi. Polyamines play a vital role in fungal growth, development, and differentiation (Valdés-Santiago et al. 2012). Preliminary tests with BIOLOG (Hayward, CA, U.S.A.) phenotypic microarrays suggest that deletion strains may not be able to utilize the polyamine putrescine as well as the WT (data not shown). This was documented by the use of BIOLOG PM2A MicroPlate carbon source, where growth of the WT strain was detectable on putrescine while the $\Delta F v s t u A$ mutants were not, suggesting that the mutants were unable to metabolize putrescine. To the best of our knowledge, this investigation is the first suggestion of regulation of polyamines by stuA in Fusarium spp.

The $\Delta F v s t u A$-deletion strains $\Delta F v s t u A 1 / 2$ and $\Delta F v s t u A 1 / 7$ were significantly less virulent on maize seedlings than the WT strain. However, seedlings inoculated with $\Delta F v s t u A 2 / 8$ did not differ from WT in either height or weight (Fig. 7). Since the $\Delta F v s t u A$ mutants were reduced in mycelial growth and conidiation, the reduced virulence of the mutants may be related to their developmental growth defects. However, studies have also reported $F$. verticillioides mutants that have impaired vegetative growth but are not decreased in virulence (Choi and $\mathrm{Xu}$ 2010; Sagaram et al. 2007). Additionally, the relatively slow growth of $\Delta F v s t u A 2 / 8$ but higher degree of virulence compared with the other two $\triangle F v s t u A$ mutants supports the assertion that in-vitro growth of the $\Delta F v s t u A$ mutants is not the main driver in terms of the variation in virulence in these mutants. Consequently, the observed reduction in virulence is likely due to a direct role of FvSTUA in the infection process. Additionally, the ability of one of the $\Delta F v s t u A$ mutants to retain or regain its virulence suggests the potential role of second-site suppressor mutations or other unknown influencing factors. Fumonisin is a known virulence and pathogenicity factor of $F$. verticillioides, particularly in maize seedling blight disease (Glenn et al. 2008). Toxin production and pathogenicity are interlinked in $F$. graminearum (Jansen et al. 2005), and loss of tricothecene production in FgStuA mutants was a major factor in their reduced pathogenicity (Lysøe et al. 2011). The same may well occur in $F$. verticillioides on some maize genotypes.

In this study, fumonisin production by $\Delta F v s t u A$ mutants in GYAM medium was reduced significantly for all tested mutants (Fig. 6), and the expression of several FUM genes decreased significantly in the $\Delta F v s t u A$ strains. These findings indicate that FvSTUA positively regulates fumonisin biosynthesis. Deletion of FvSTUA also suppressed expression of the FUS cluster dramatically, which indicates positive regulation of fusarin $\mathrm{C}$ biosynthesis by FvSTUA in GYAM medium. The contrasting expression of the $F U B$ cluster, which encodes enzymes for fusaric acid biosynthesis, in the different mutants makes it difficult to determine the effect of FvSTUA on fusaric acid production. However, it is clear that FvSTUA impacts regulation of fusaric acid. The reduced toxin production by $\Delta F v s t u A$ mutants could partly explain the loss of virulence observed in maize seedlings. But, as noted above, it is not the only reason for the loss of virulence, which is supported by the reduced fumonisin production of $\Delta F v s t u A 2 / 8$ but WT virulence. Further research is needed to understand the mechanisms by which FvSTUA regulates biosynthesis of fumonisins and other secondary metabolites. The low production of fumonisin by hyg-resistant progeny, similar to that of the parental $\Delta F v s t u A$ mutants and the relatively high production of fumonisin by hyg-sensitive progeny (Fig. 6), indicates that the phenotypes associated with $F v s t u A$ mutation are heritable. This is also supported by the reduced conidiation of the hyg-resistant progeny and WT conidiation levels by hyg-sensitive progeny (data not shown).

This study provides evidence of the role of FvSTUA in various fundamental biological processes in $F$. verticillioides. Like other fungal species, $\Delta F v s t u A$-deletion strains exhibit radically different morphologies from the WT (i.e., stunted aerial hyphae and reduced macro- and microconidiation). In conditions conducive to toxin production, the ability of mutants to produce fumonisins was significantly reduced. Gene expression studies provide evidence of FvSTUA regulation of not only fumonisin but fusarin $\mathrm{C}$ and fusaric acid biosynthetic clusters as well. It would be interesting to study the genetic network of these genes to understand their process of regulation. We interpret much of the phenotypic and gene expression variation within the $\Delta F v s t u A$ deletion strains examined to likely be the result of secondary compensatory genome alterations within the individual strains. As indicated, sectoring with these mutants is common compared with WT. This suggests that the deletion of FvSTUA is destructive to the operation of the cells. We hypothesize, based on the phenotypic and gene expression variation, that each of the examined $\Delta F v s t u A$ strains is working out its own genetic/physiological strategy of compensation for the defect in transcriptional control of important developmental genes impacting diverse cellular processes. Future work beyond the scope of this report could involve whole-genome sequencing of the deletion strains that were analyzed by RNA-Seq. This approach should allow identification of second-site compensatory mutations. 


\section{MATERIALS AND METHODS}

Strains and culture methods.

We used Fusarium verticillioides WT strain FRC M-3125 (here referred to as M3125, the same as the sequenced strain referred to as 7600 [FungiDB; Stajich et al. 2012]) and $\Delta$ FvstuA mutants generated from M3125. Fungal strains, primers, and plamids used in this study are listed in Table 4. Fungal strains were stored long-term in glycerol at $-80^{\circ} \mathrm{C}$ and were maintained as working stocks on PDA (Acumedia) and PDA containing $150 \mu \mathrm{g}$ of hygromycin B per millimiter (InvivoGen) plates as needed.

\section{Targeted FvSTUA deletion.}

To study the function of $F v S T U A$, the gene was deleted using the one-step construction of Agrobacterium-recombination ready-plasmids (OSCAR) (Paz et al. 2011) method and subsequent ATMT of $F$. verticillioides with the deletion construct (Kang et al. 2005; Mullins et al. 2001). Briefly, to create the pOSCAR-FvSTUA plasmid (FVEG_02853_OSC_NJC_13), we used the primers FVEG_02853 O1-O4 to replace 1,767 bp of the FvSTUA coding region with the hygromycin-resistance selection marker from p-A-HYG-OSCAR, flanked by $1,319 \mathrm{bp}$ of 5' and 1,459 bp of $3^{\prime} F v S T U A$ regions. We performed ATMT using a previously described protocol (Khang et al. 2005). pOSCAR-FvSTUA-transformed A. tumefaciens was grown at $28^{\circ} \mathrm{C}$ for 2 days in $5 \mathrm{ml}$ of minimal medium, supplemented with spectinomycin $(100 \mu \mathrm{g} / \mathrm{ml})$ and then diluted to an optical density at $600 \mathrm{~nm}\left(\mathrm{OD}_{600}\right)=0.15$ in induction medium containing acetosyringone and spectinomycin, and was grown for 2 days at $28^{\circ} \mathrm{C}$. For cocultivation, freshly harvested $F$. verticillioides conidia were used. After mixing equal volumes of a $F$. verticillioides conidial suspension $\left(10^{6}\right.$ conidia/ml $)$ and induced Agrobacterium tumefaciens cells grown to $\mathrm{OD}_{600}=0.6$, we spread $200 \mu \mathrm{l}$ of the mix on sterilized 47-mm mixed ester cellulose membrane filters (Thermo Fisher Scientific) on cocultivation medium. Following cocultivation at $27^{\circ} \mathrm{C}$ for $48 \mathrm{~h}$, the membranes were transferred onto Aspergillus minimal medium (Khang et al. 2005) amended with $150 \mu \mathrm{g}$ of hygromycin per milliliter and $200 \mu \mathrm{M}$ cefotaxime to select transformants. Seven to 10 days later, fungal colonies grown from the membrane were transferred to PDA-hyg plates. Prospective transformants were purified as single conidial isolates and were reselected on PDA-hyg plates. Nine deletion mutants were obtained from 18 transformants. The transformants were screened by PCR to confirm the absence of the ORF. We used Southern blot hybridization by standard methods to confirm the deletion of FvSTUA. Genomic DNA from the WT M3125 and the nine $\Delta F v s t u A$ mutants was isolated using a DNeasy plant mini kit (Qiagen) and was digested with EcoRI (Promega). A mixed digoxigenin (DIG)-labeled probe was generated by PCR (primers used are listed in Table 4) containing the ORFs of both FVEG_11838 (internal positive control) and FVEG_02853 (gene of interest deleted) with the Roche DIG-High Prime DNA labeling and detection kit (Millipore Sigma), according to manufacturer instructions.

\section{Generation of $\Delta F v s t u A$ progeny.}

Because we were unable to show complementation by reintroduction of the WT FvStuA sequence (not shown), we generated progeny to study phenotypic cosegregation of the mutant phenotypes with the deletion allele as an alternative approach. Genetic crosses were performed on carrot agar to obtain progeny of $\Delta F v s t u A$ deletion strains $(\Delta F v s t u A 1 / 2, \Delta F v s t u A 1 / 7$, and $\Delta F v s t u A 2 / 8)$ via the method described by Klittich and Leslie (1988). The deletion mutants were used as male strains and the $F$. verticillioides-compatible WT strain A00999 was used as the female parent. The male strains were cultured on PDA (Acumedia) for 5 days, and the female strains were cultured on carrot agar at $27^{\circ} \mathrm{C}$ for 7 days. Male conidia isolated from the PDA plates were spread over the female mycelium for fertilization. The crosses were incubated for 3 weeks at 25 to $27^{\circ} \mathrm{C}$ with a 16 -h light and 8 -h dark cycle. Cirrhi were collected from mature perithecia and were suspended individually in $700 \mu \mathrm{l}$ of sterile water. Fifty-microliter aliquots of the resulting ascospore suspension were plated onto $3 \%$ water agar (wt/vol). The plates were incubated for roughly 2 days, and single germinating ascospores were transferred to PDA slants. At least one cirrhus was collected from each cross, and $\leq 24$ germinating

Table 4. Strains, plasmids, and primers used in this study

\begin{tabular}{|c|c|}
\hline Name & Genotype, source, or sequence $\left(5^{\prime} \rightarrow 3^{\prime}\right)$ \\
\hline \multicolumn{2}{|l|}{ Strain } \\
\hline WT & Fusarium verticillioides wild-type strain FRC M3125 (7600) \\
\hline$\Delta F v s t u A 1 / 2$ & $F$. verticillioides $F v S T U A$ deletion strain \\
\hline$\Delta F v s t u A 1 / 7$ & F. verticillioides FvSTUA deletion strain \\
\hline$\Delta F v s t u A 2 / 8$ & F. verticillioides FvSTUA deletion strain \\
\hline$\Delta F v s t u A 2 / 11$ & F. verticillioides $F v S T U A$ deletion strain \\
\hline$\Delta F v s t u A 2 / 12$ & $F$ verticillioides $F v S T U A$ deletion strain \\
\hline FvSTUA-tdTomato 2.1-1 & F. verticillioides strain transformed with FvSTUA-tdTomato \\
\hline \multicolumn{2}{|l|}{ Plasmid } \\
\hline pBV1 & C.-H. Khang, Plant Biology, University of Georgia \\
\hline pBV359 & C.-H. Khang, Plant Biology, University of Georgia \\
\hline \multicolumn{2}{|l|}{ Primers } \\
\hline StuA-gfp-Rf & GGGCCCGGATCCATCTATCAG \\
\hline StuA-gfp-Ff & GAGAGAGAATTCTGGGTGCACAGTAACTGC \\
\hline FVEG_00317 (FUM 6)-Rev & CACAGCCCTATCTCCGTTCTTTAC \\
\hline FVEG_02853_O1 & GGGGACAGCTTTCTTGTACAAAGTGGAAAGCAGGTACCCAAGCATTGAGTCT \\
\hline FVEG_02853_O2 & GGGGACTGCTTTTTTGTACAAACTTGTGCACAATTGCGAAGAGGAAGCAGT \\
\hline FVEG_02853_O3 & GGGGACAACTTTGTATAGAAAAGTTGTTTGGAAAGAGTCTCTGCAGCAAGGT \\
\hline FVEG_02853_O4 & GGGGACAACTTTGTATAATAAAGTTGTATGCAGTATCGTTGAGGGCAGACA \\
\hline 5/1 (AMMM $\left.{ }^{\mathrm{a}}\right)$ FVEG_02853 & TCTGTTGTGCGTTGACAGGAGAGT \\
\hline FVEG_02853_ORF_For & AAT GCA GCA GCC AGG TTA CAG T \\
\hline FVEG_02853_ORF_Rev & TGC TTC GAA GTT GCC TGC AGA A \\
\hline FVEG_11838_ORF_For & ATG GCA GAA CCA ACT GTC CAC \\
\hline FVEG_11838_ORF_Rev & AAT CAA TCG AGG CGC AGC AA \\
\hline Hyg_Rev & GCCGATGCAAAGTGCCGATAAACA \\
\hline
\end{tabular}

\footnotetext{
${ }^{\mathrm{a}} \mathrm{AMM}=$ Aspergillus minimal medium.
} 
ascospores were collected per perithecium (Leslie 1991). After 4 to 7 days of growth, the progeny were transferred onto PDA and PDA-hyg to screen for hygromycin-resistant and -sensitive strains. Hygromycin B-resistant and -sensitive strains were replated on PDA-hyg and PDA, respectively, to use for further studies and long-term storage.

\section{Construction of FvstuA-tdTomato translational fusion vectors.}

The plasmid pBV1 (pBHt2) (Mullins et al. 2001) (a gift from C.-H. Khang, University of Georgia) was digested with EcoRI and HindIII to generate a plasmid backbone with the hyg selection marker driven by the TrpC promoter from Aspergillus nidulans. The FvSTUA gene along with its promoter was amplified from $F$. verticillioides genomic DNA with primers adding $5^{\prime}$ and $3^{\prime}$ EcoRI and BamHI restriction sites, respectively. The DNA fragment containing the tdTomato protein-encoding gene and terminator was excised from pBV359 (also a gift from C.-H. Khang) with BamHI and HindIII. The pBV1 plasmid backbone, the DNA fragments with FvSTUA, and the DNA fragment with the tdTomato gene were individually electrophoresed on a $0.7 \%$ low-melting-point agarose gel, were excised, and were subjected to a low-melting-point agarose ligation (Crouse et al. 1983), which resulted in plasmid pFvSTUA-tdTomato. The resulting expressed protein is a C-terminal FvSTUA-tdTomato fusion driven by the native $F v S T U A$ promoter. pFvSTUA-tdTomato was finally introduced into M3125 by ATMT. Hyphae of three randomly chosen FvSTUA-tdTomato transformants (FvSTUAtdTomato2.1-1, 2.2-3, 2.4-2) were visualized, using a Zeiss Axio Imager.A2 microscope (Zeiss). Images were captured using an AxioCam 415 ERc 5s camera and were processed using Zeiss Zen software. A Lumen Dynamics X-Cite 120Q light source (Excelitas Technology Corp.) with Zeiss filter set 20 (rhodamine; for td-Tomato) was used to observe fluorescence.

\section{Vegetative growth and micro- and macroconidiation assays.}

Petri plates $(100 \mathrm{~mm})$ containing PDA were inoculated with fungal strains from $-80^{\circ} \mathrm{C}$ glycerol stocks. Cork borer plugs ( $7 \mathrm{~mm})$ were transferred from these starter plate cultures to $100-\mathrm{mm}$ PDA plates and were grown at $27^{\circ} \mathrm{C}$ in the dark. These plate cultures were used in the tests described below.

For the growth assays, radial growth (diameter) was measured 5 and 10 days postinoculation (dpi), with three replicates. For microconidiation, two $7-\mathrm{mm}$ plugs were taken at $5 \mathrm{dpi}$ from each side of two perpendicular diameters drawn on the plate (a total of eight plugs) (Supplementary Fig. S4) and were transferred to $3 \mathrm{ml}$ of sterilized water in 14-ml snap-cap tubes (Falcon) and were shaken for $15 \mathrm{~min}$ at $25^{\circ} \mathrm{C}$. Conidial suspension $(1 \mathrm{ml})$ was transferred to a microfuge tube for spore counting. The same procedure was followed for the 10-dpi cultures, except three plugs were taken from each side (a total of 12 plugs) (Supplementary Fig. S4). A hemocytometer was used to count conidia for the first repeat and an automated cell counter (Cellometer Vision CBA, Nexcelom Bioscience) was used for the final two repeats. To test for macroconidiation, cultures were grown on $\mathrm{CMC}$ containing $15 \mathrm{~g}$ of carboxymethylcellulose, $1 \mathrm{~g}$ of $\mathrm{NH}_{4} \mathrm{NO}_{3}, 1 \mathrm{~g}$ of $\mathrm{KH}_{2} \mathrm{PO}_{4}, 1 \mathrm{~g}$ of yeast extract, $0.5 \mathrm{~g}$ of $\mathrm{MgSO}_{4} \cdot 7 \mathrm{H}_{2} \mathrm{O}$, and $1.5 \%$ agar per liter of water. The strains were grown on $\mathrm{CMC}$ plates at $27^{\circ} \mathrm{C}$ in the dark and were microscopically observed and photographed as noted above. Test Petri plates were $100 \mathrm{~mm}$ in diameter with a standard volume of $18 \mathrm{ml}$ of medium, poured with PourMatic MP-1000 (New Brunswick Scientific). Each test was conducted in triplicate and each strain had three replicates in each assay. To test surface hydrophobicity, a $10-\mu l$ droplet of distilled water was added to the top of 5-dayold fungal colonies grown on PDA, and the persistence of the water droplet was examined over time. Growth and microconidiation assays for progeny of deletion strains was performed as described above.

\section{Fumonisin analysis.}

Assesment of fumonisin production was conducted in GYAM medium (Brown et al. 2015; Proctor et al. 1999), containing $0.05 \%$ yeast extract, $0.24 \mathrm{M}$ glucose, $8 \mathrm{mM}$ L-asparagine, $1.7 \mathrm{mM} \mathrm{NaCl}, 4.4 \mathrm{mM} \mathrm{K}_{2} \mathrm{HPO}_{4}, 2 \mathrm{mM} \mathrm{MgSO}_{4}, 8.8 \mathrm{mM} \mathrm{CaCl}_{2}$, and $5.0 \mathrm{mM}$ malic acid. Fifty microliters of a $2 \times 10^{6}$ conidia $/ \mathrm{ml}$ suspension of each strain was added to $1,950 \mu \mathrm{l}$ of GYAM medium in a sterile snap-cap tube (final concentration of $5 \times 10^{4}$ conidia/ml) and were grown with the caps unsnapped at $27^{\circ} \mathrm{C}$ at $225 \mathrm{rpm}$ in the dark for 7 days. Two milliliters of acetonitrile + $5 \%$ formic acid was added to the tubes, was mixed, and was allowed to stand for $30 \mathrm{~min}$. The mixture was vacuum-filtered onto previously weighed sterile 25-mm, 5-micron pore size MAGNA nylon filters (MSI), which were stored in a desiccator under vacuum for $\geq 24 \mathrm{~h}$ prior to obtaining the tare weight. The mycelial mass on the filter paper was allowed to dry for $\geq 24 \mathrm{~h}$ in a vacuum dessicator and was then weighed to obtain the biomass for each strain. The filtered lysate (roughly $3 \mathrm{ml}$ ) was diluted (1:1) via the addition of $3 \mathrm{ml}$ of distilled water. The diluted samples were then analyzed for fumonisin content as described by Williams et al. (2007), using high performance liquid chromatography-mass spectrometry.

\section{Virulence assays.}

Virulence assays of the maize seedlings were carried out as described previously (Glenn et al. 2008), with some minor modifications. Briefly, Silver Queen seeds (W. Atlee Burpee \& Co.) were rinsed in sterile deionized water, were surfacesterilized for $10 \mathrm{~min}$ in $5.25 \%$ sodium hypochlorite, were rinsed four times with sterile water, and were soaked for $4 \mathrm{~h}$ in sterile water. The seeds were then heat-treated for $5 \mathrm{~min}$ at $60^{\circ} \mathrm{C}$ and were rinsed twice with room temperature sterilized water. For each fungal strain examined, 40 sterilized seeds were placed in 100 -mm Petri plates and were flooded with $10 \mathrm{ml}$ of the appropriate $10^{4}$-conidia/ml conidial suspension. Sterilized deionized water was used for the control seeds. The seeds were then incubated overnight at $27^{\circ} \mathrm{C}$ in the dark. For each strain and treatment, three replicates of 10 germinated seeds each were planted in a twice-autoclaved growth mix (2M Mix; Conrad Fafard Inc.) in 4inch plastic pots sterilized by autoclaving. The pots were placed in a growth room with a 14-h light cycle at $30^{\circ} \mathrm{C}$ (an average of $254 \mu \mathrm{mol} \mathrm{m} \mathrm{m}^{-2} \mathrm{~s}^{-1}$ ) and a 10 -h dark cycle at $20^{\circ} \mathrm{C}$. The shoots were harvested above the soil line with a sterile razorblade 14 dap, and the length and fresh weight of each shoot was measured.

\section{Transcriptome analysis.}

Transcriptome analysis was performed for WT M3125 and three independent deletion mutants $(\Delta F v s t u A 1 / 2, \Delta F v s t u A 1 / 7$, and $\Delta F v s t u A 2 / 8$ ), cultured in both PDB and GYAM. To generate a final spore concentration of $5 \times 10^{4}$ conidia $/ \mathrm{ml}, 40 \mu \mathrm{l}$ of $2.5 \times 10^{6}$ conidia $/ \mathrm{ml}$ of each strain was added to $1.96 \mathrm{ml}$ of PDB medium in sterile 14-ml snap-cap tubes and were grown at $27^{\circ} \mathrm{C}$ at $225 \mathrm{rpm}$ in the dark for $30 \mathrm{~h}$, with the caps unsnapped. Liquid well-mixed culture $(1 \mathrm{ml})$ was added to a $\mathrm{Z}$ matrix extraction tube (MP Biomedicals) and was centrifuged at 10,000 $\times g$ for $5 \mathrm{~min}$ at $4^{\circ} \mathrm{C}$. After discarding the supernatant, $1 \mathrm{ml}$ of lysis buffer (PureLink RNA kit, ThermoFischer Scientific) was added to the fungal pellet. The pellet was then homogenized in a Fast Prep-24 5G (MP Biomedicals) twice at $6 \mathrm{~m}$ per second for $30 \mathrm{~s}$, with a 1-min rest between pulses. The lysate was transferred to a homogenizer tube (PureLink RNA mini kit; ThermoFischer Scientific), and total RNA extraction was conducted, per manufacturer instructions, with On-column PureLink DNase treatment. The quality of RNA samples was determined using a RNA 6000 
Nano kit on an Agilent 2100 Bioanalyzer (Agilent Technologies). The libraries were made with the Illumina TruSeq Stranded mRNA library prep kit (Illumina), as per manufacturer instructions, and the pooled libraries were pair-end sequenced at the Georgia Genomics Facility (GGF) (Athens, GA, U.S.A.), using an Illumina NextSeq 500 sequencer (Illumina).

To isolate total RNA from $F$. verticillioides, cultures were grown in GYAM medium, were harvested, and total RNA was extracted as noted above. However, library preparations and sequencing were performed by the GGF and the library was made using a KAPA Stranded mRNA-Seq kit (Kapa Biosystems), according to the manufacturer instructions.

RNA sequence data analysis was done with the suite of tools on the Galaxy server at the University of Georgia. The sequence data were mapped to the $F$. verticillioides reference genome (FungiDB, release 31, genome release date 2016-10-12) with TopHat. Gene expression was quantified, via Cufflinks, as fragments per kilobase per million mapped reads. Cuffdiff was used to obtain differentially expressed genes between M3125 and $\Delta F v s t u A$ strains. Differentially expressed genes were analyzed using FungiDB and Blast2GO (version 4.0). The generated data set was deposited at the National Center for Biotechnology under the accession GSE144966.

\section{Statistical analysis.}

We performed statistical analyses using SAS System (SAS University Edition; SAS Institute). Treatments for the different assays were evaluated for significance by ANOVA followed by comparison of least square means.

\section{ACKNOWLEDGMENTS}

We thank T. Mitchell for assistance with fumonisin quantification.

\section{AUTHOR-RECOMMENDED INTERNET RESOURCE}

FungiDB: https://www.fungidb.org

\section{LITERATURE CITED}

Abel, S., and Gelderblom, W. C. 1998. Oxidative damage and fumonisin $\mathrm{B}_{1}$-induced toxicity in primary rat hepatocytes and rat liver in vivo. Toxicology 131:121-131.

Aramayo, R., Peleg, Y., Addison, R., and Metzenberg, R. 1996. Asm-1 ${ }^{+}$, a Neurospora crassa gene related to transcriptional regulators of fungal development. Genetics 144:991-1003.

Bacon, C. W., Glenn, A. E., and Yates, I. E. 2008. Fusarium verticillioides: Managing the endophytic association with maize for reduced fumonisins accumulation. Toxin Rev. 27:411-446.

Bacon, C. W., and Hinton, D. M. 1996. Symptomless endophytic colonization of maize by Fusarium moniliforme. Can. J. Bot. 74:1195-1202.

Bacon, C. W., Yates, I. E., Hinton, D. M., and Meredith, F. 2001. Biological control of Fusarium moniliforme in maize. Environ. Health Perspect. 109 (Suppl 2):325-332.

Baeza-Montañez, L., Gold, S. E., Espeso, E. A., and García-Pedrajas, M. D. 2015. Conserved and distinct functions of the "stunted" (StuA)-homolog Ust1 during cell differentiation in the corn smut fungus Ustilago maydis. Mol. Plant-Microbe Interact. 28:86-102.

Bailey, L. A., and Ebbole, D. J. 1998. The fluffy gene of Neurospora crassa encodes a Gal4p-type C6 zinc cluster protein required for conidial development. Genetics 148:1813-1820.

Brown, D. W., Butchko, R. A., Busman, M., and Proctor, R. H. 2012 Identification of gene clusters associated with fusaric acid, fusarin, and perithecial pigment production in Fusarium verticillioides. Fungal Genet. Biol. 49:521-532.

Brown, D. W., Lee, S. H., Kim, L. H., Ryu, J. G., Lee, S., Seo, Y., Kim, Y. H., Busman, M., Yun, S. H., Proctor, R. H., and Lee, T. 2015. Identification of a 12gene fusaric acid biosynthetic gene cluster in Fusarium species through comparative and functional genomics. Mol. Plant-Microbe Interact. 28:319-332.

Choi, Y. E., and Shim, W. B. 2008. Functional characterization of Fusarium verticillioides $C P P 1$, a gene encoding a putative protein phosphatase $2 \mathrm{~A}$ catalytic subunit. Microbiology 154:326-336.
Choi, Y. E., and Xu, J. R. 2010. The cAMP signaling pathway in Fusarium verticillioides is important for conidiation, plant infection, and stress responses but not fumonisin production. Mol. Plant-Microbe Interact. 23:522-533

Clutterbuck, A. J. 1969. A mutational analysis of conidial development in Aspergillus nidulans. Genetics 63:317-327.

Crouse, G. F., Frischauf, A., and Lehrach, H. 1983. An integrated and simplified approach to cloning into plasmids and single-stranded phages. Methods Enzymol. 101:78-89.

Desjardins, A. E. 2003. Gibberella from A (venaceae) to $Z$ (eae). Annu. Rev. Phytopathol. 41:177-198.

Dutton, J. R., Johns, S., and Miller, B. L. 1997. StuAp is a sequence-specific transcription factor that regulates developmental complexity in Aspergillus nidulans. EMBO J. 16:5710-5721.

Foley, D. C. 1962. Systemic infection of corn by Fusarium moniliforme. Phytopathology 52:870-872.

Fuchs, U., Czymmek, K. J., and Sweigard, J. A. 2004. Five hydrophobin genes in Fusarium verticillioides include two required for microconidial chain formation. Fungal Genet. Biol. 41:852-864.

Gao, S., Gold, S. E., and Glenn, A. E. 2018. Characterization of two catalase-peroxidase-encoding genes in Fusarium verticillioides reveals differential responses to in vitro versus in planta oxidative challenges. Mol. Plant Pathol. 19:1127-1139.

García-Pedrajas, M. D., Baeza-Montañez, L., and Gold, S. E. 2010. Regulation of Ustilago maydis dimorphism, sporulation, and pathogenic development by a transcription factor with a highly conserved APSES domain. Mol. Plant-Microbe Interact. 23:211-222.

Gatch, E. W., and Munkvold, G. P. 2002. Fungal species composition in maize stalks in relation to European corn borer injury and transgenic insect protection. Plant Dis. 86:1156-1162.

Giles, S. S., Stajich, J. E., Nichols, C., Gerrald, Q. D., Alspaugh, J. A., Dietrich, F., and Perfect, J. R. 2006. The Cryptococcus neoformans catalase gene family and its role in antioxidant defense. Eukaryot. Cell 5: 1447-1459.

Gimeno, C. J., and Fink, G. R. 1994. Induction of pseudohyphal growth by overexpression of PHD1, a Saccharomyces cerevisiae gene related to transcriptional regulators of fungal development. Mol. Cell. Biol 14:2100-2112.

Glenn, A. E. 2006. Natural variation of ascospore and conidial germination by Fusarium verticillioides and other Fusarium species. Mycol. Res. 110:211-219.

Glenn, A. E., Richardson, E. A., and Bacon, C. W. 2004. Genetic and morphological characterization of a Fusarium verticillioides conidiation mutant. Mycologia 96:968-980.

Glenn, A. E., Zitomer, N. C., Zimeri, A. M., Williams, L. D., Riley, R. T., and Proctor, R. H. 2008. Transformation-mediated complementation of a FUM gene cluster deletion in Fusarium verticillioides restores both fumonisin production and pathogenicity on maize seedlings. Mol. PlantMicrobe Interact. 21:87-97.

Guo, L., Wenner, N., and Kuldau, G. A. 2015. FvSO regulates vegetative hyphal fusion, asexual growth, fumonisin B1 production, and virulence in Fusarium verticillioides. Fungal Biol. 119:1158-1169.

IpCho, S. V., Tan, K. C., Koh, G., Gummer, J., Oliver, R. P., Trengove, R. D., and Solomon, P. S. 2010. The transcription factor StuA regulates central carbon metabolism, mycotoxin production, and effector gene expression in the wheat pathogen Stagonospora nodorum. Eukaryot. Cell 9:1100-1108

Islamovic, E., García-Pedrajas, M. D., Chacko, N., Andrews, D. L., Covert, S. F., and Gold, S. E. 2015. Transcriptome analysis of a Ustilago maydis ust 1 deletion mutant uncovers involvement of laccase and polyketide synthase genes in spore development. Mol. Plant-Microbe Interact. 28: 42-54.

Jansen, C., von Wettstein, D., Schäfer, W., Kogel, K. H., Felk, A., and Maier, F. J. 2005. Infection patterns in barley and wheat spikes inoculated with wild-type and trichodiene synthase gene disrupted Fusarium graminearum. Proc. Natl. Acad. Sci. U.S.A. 102:16892-16897.

Kershaw, M. J., and Talbot, N. J. 1998. Hydrophobins and repellents: Proteins with fundamental roles in fungal morphogenesis. Fungal Genet. Biol. 23:18-33.

Khang, C. H., Park, S. Y., Lee, Y. H., and Kang, S. 2005. A dual selection based, targeted gene replacement tool for Magnaporthe grisea and Fusarium oxysporum. Fungal Genet. Biol. 42:483-492.

Klittich, C., and Leslie, J. F. 1988. Nitrate reduction mutants of Fusarium moniliforme (Gibberella fujikuroi). Genetics 118:417-423.

Leslie, J. F. 1991. Mating populations in Gibberella fujikuroi (Fusarium section Liseola). Phytopathology 81:1058-1060.

Leslie, J. F., and Summerell, B. A. 2006. The Fusarium Laboratory Manual. Blackwell Publishing, Hoboken, NJ, U.S.A. 
Li, S., Myung, K., Guse, D., Donkin, B., Proctor, R. H., Grayburn, W. S., and Calvo, A. M. 2006. FvVE1 regulates filamentous growth, the ratio of microconidia to macroconidia and cell wall formation in Fusarium verticillioides. Mol. Microbiol. 62:1418-1432.

Lysøe, E., Pasquali, M., Breakspear, A., and Kistler, H. C. 2011. The transcription factor FgStuAp influences spore development, pathogenicity, and secondary metabolism in Fusarium graminearum. Mol. PlantMicrobe Interact. 24:54-67.

Malapi-Wight, M., Kim, J. E., and Shim, W. B. 2014. The N-terminus region of the putative $\mathrm{C}_{2} \mathrm{H}_{2}$ transcription factor Ada1 harbors a speciesspecific activation motif that regulates asexual reproduction in Fusarium verticillioides. Fungal Genet. Biol. 62:25-33.

Marasas, W. F. 2001. Discovery and occurrence of the fumonisins: A historical perspective. Environ. Health Perspect. 109 (Suppl 2): 239-243.

Miller, K. Y., Toennis, T. M., Adams, T. H., and Miller, B. L. 1991. Isolation and transcriptional characterization of a morphological modifier: The Aspergillus nidulans stunted (stuA) gene. Mol. Gen. Genet. 227:285-292.

Miller, K. Y., Wu, J., and Miller, B. L. 1992. StuA is required for cell pattern formation in Aspergillus. Genes Dev. 6:1770-1782.

Mullins, E. D., Chen, X., Romaine, P., Raina, R., Geiser, D. M., and Kang, S. 2001. Agrobacterium-mediated transformation of Fusarium oxysporum: An efficient tool for insertional mutagenesis and gene transfer. Phytopathology 91:173-180.

Munkvold, G. P., and Desjardins, A. E. 1997. Fumonisins in maize: Can we reduce their occurrence? Plant Dis. 81:556-565.

Munkvold, G. P., Hellmich, R. L., and Showers, W. B. 1997. Reduced fusarium ear rot and symptomless infection in kernels of maize genetically engineered for European corn borer resistance. Phytopathology 87:1071-1077.

Musser, S. M., and Plattner, R. D. 1997. Fumonisin composition in cultures of Fusarium moniliforme, Fusarium proliferatum, and Fusarium nygami. J. Agric. Food Chem. 45:1169-1173.

Navarro, R. E., Stringer, M. A., Hansberg, W., Timberlake, W. E., and Aguirre, J. 1996. catA, a new Aspergillus nidulans gene encoding a developmentally regulated catalase. Curr. Genet. 29:352-359.

Nelson, P. E., Toussoun, T. A., and Marasas, W. F. 1983. Fusarium species: An illustrated manual for identification. Pennsylvania State University Press, University Park, PA, U.S.A.

Nishimura, M., Fukada, J., Moriwaki, A., Fujikawa, T., Ohashi, M., Hibi, T., and Hayashi, N. 2009. Mstu1, an APSES transcription factor, is required for appressorium-mediated infection in Magnaporthe grisea. Biosci. Biotechnol. Biochem. 73:1779-1786.

Niu, C., Payne, G. A., and Woloshuk, C. P. 2015. Transcriptome changes in Fusarium verticillioides caused by mutation in the transporter-like gene FST1. BMC Microbiol. 15:90.

Ohara, T., and Tsuge, T. 2004. FoSTUA, encoding a basic helix-loop-helix protein, differentially regulates development of three kinds of asexual spores, macroconidia, microconidia, and chlamydospores, in the fungal plant pathogen Fusarium oxysporum. Eukaryot. Cell 3:1412-1422.

Ortiz, C. S., and Shim, W. B. 2013. The role of MADS-box transcription factors in secondary metabolism and sexual development in the maize pathogen Fusarium verticillioides. Microbiology 159:2259-2268.

Paz, Z., García-Pedrajas, M. D., Andrews, D. L., Klosterman, S. J., Baeza-Montañez, L., and Gold, S. E. 2011. One step construction of Agrobacterium-recombination-ready-plasmids (OSCAR), an efficient and robust tool for ATMT based gene deletion construction in fungi. Fungal Genet. Biol. 48:677-684.

Pitt, J. I., Wild, C. P., Baan, R. A., Gelderblom, W. C. A., Miller, J. D., Riley, R. T., and Wu, F. 2012. Mycotoxins and human health. Pages 87-104 in: Improving Public Health Through Mycotoxin Control. IARC Scientific Publications. No. 158, World Health Organization, Lyon, France.

Proctor, R. H., Desjardins, A. E., Plattner, R. D., and Hohn, T. M. 1999. A polyketide synthase gene required for biosynthesis of fumonisin mycotoxins in Gibberella fujikuroi mating population. A. Fungal Genet. Biol. 27:100-112.

Ramírez-Zavala, B., and Domínguez, A. 2008. Evolution and phylogenetic relationships of APSES proteins from Hemiascomycetes. FEMS Yeast Res. 8:511-519.

Rheeder, J. P., Marasas, W. F., Thiel, P. G., Sydenham, E. W., Shephard, G. S., and Van Schalkwyk, D. J. 1992. Fusarium moniliforme and fumonisins in corn in relation to human esophageal cancer in Transkei. Phytopathology 82:353-357.

Ridenour, J. B., and Bluhm, B. H. 2014. The HAP complex in Fusarium verticillioides is a key regulator of growth, morphogenesis, secondary metabolism, and pathogenesis. Fungal Genet. Biol. 69:52-64.

Ridenour, J. B., Smith, J. E., Hirsch, R. L., Horevaj, P., Kim, H., Sharma, S., and Bluhm, B. H. 2014. UBL1 of Fusarium verticillioides links the $\mathrm{N}$-end rule pathway to extracellular sensing and plant pathogenesis. Environ. Microbiol. 16:2004-2022.
Sagaram, U. S., Shaw, B. D., and Shim, W. B. 2007. Fusarium verticillioides $G A P 1$, a gene encoding a putative glycolipid-anchored surface protein participates in conidiation and cell wall structure but not virulence. Microbiology 153:2850-2861.

Scherer, M., Wei, H., Liese, R., and Fischer, R. 2002. Aspergillus nidulans catalase-peroxidase gene (cpeA) is transcriptionally induced during sexual development through the transcription factor StuA. Eukaryot. Cell 1:725-735.

Shephard, G. S., Thiel, P. G., Stockenström, S., and Sydenham, E. W. 1996. Worldwide survey of fumonisin contamination of corn and corn-based products. J. AOAC Int. 79:671-687.

Shim, W. B., and Woloshuk, C. P. 2001. Regulation of fumonisin $B_{1}$ biosynthesis and conidiation in Fusarium verticillioides by a cyclin-like (C-type) gene, FCC1. Appl. Environ. Microbiol. 67:1607-1612.

Shin, J. H., Kim, J. E., Malapi-Wight, M., Choi, Y. E., Shaw, B. D., and Shim, W. B. 2013. Protein phosphatase 2A regulatory subunits perform distinct functional roles in the maize pathogen Fusarium verticillioides. Mol. Plant Pathol. 14:518-529.

Skamnioti, P., Henderson, C., Zhang, Z., Robinson, Z., and Gurr, S. J. 2007 A novel role for catalase $B$ in the maintenance of fungal cell-wall integrity during host invasion in the rice blast fungus Magnaporthe grisea. Mol. Plant-Microbe Interact. 20:568-580.

Stajich, J. E., Harris, T., Brunk, B. P., Brestelli, J., Fischer, S., Harb, O. S., Kissinger, J. C., Li, W., Nayak, V., Pinney, D. F., Stoeckert, C. J., Jr., and Roos, D. S. 2012. FungiDB: An integrated functional genomics database for fungi. Nucleic Acids Res. 40 (D1):D675-D681.

Stoldt, V. R., Sonneborn, A., Leuker, C. E., and Ernst, J. F. 1997. Efg1p, an essential regulator of morphogenesis of the human pathogen Candida albicans, is a member of a conserved class of bHLH proteins regulating morphogenetic processes in fungi. EMBO J. 16: 1982-1991.

Teertstra, W. R., Deelstra, H. J., Vranes, M., Bohlmann, R., Kahmann, R., Kämper, J., and Wösten, H. A. B. 2006. Repellents have functionally replaced hydrophobins in mediating attachment to a hydrophobic surface and in formation of hydrophobic aerial hyphae in Ustilago maydis. Microbiology 152:3607-3612.

Tong, X., Zhang, X., Plummer, K. M., Stowell, K. M., Sullivan, P. A., and Farley, P. C. 2007. GcSTUA, an APSES transcription factor, is required for generation of appressorial turgor pressure and full pathogenicity of Glomerella cingulata. Mol. Plant-Microbe Interact. 20:1102-1111.

Twumasi-Boateng, K., Yu, Y., Chen, D., Gravelat, F. N., Nierman, W. C., and Sheppard, D. C. 2009. Transcriptional profiling identifies a role for $\mathrm{BrlA}$ in the response to nitrogen depletion and for StuA in the regulation of secondary metabolite clusters in Aspergillus fumigatus. Eukaryot. Cell 8:104-115.

Urban, M., Cuzick, A., Rutherford, K., Irvine, A., Pedro, H., Pant, R., Sadanadan, V., Khamari, L., Billal, S., Mohanty, S., and HammondKosack, K. E. 2017. PHI-base: A new interface and further additions for the multi-species pathogen-host interactions database. Nucleic Acids Res. 45 (D1):D604-D610.

Valdés-Santiago, L., Cervantes-Chávez, J. A., León-Ramírez, C. G., and Ruiz-Herrera, J. 2012. Polyamine metabolism in fungi with emphasis on phytopathogenic species. J. Amino Acids 2012:837932.

Ward, M. P., Gimeno, C. J., Fink, G. R., and Garrett, S. 1995. SOK2 may regulate cyclic AMP-dependent protein kinase-stimulated growth and pseudohyphal development by repressing transcription. Mol. Cell. Biol. 15:6854-6863.

White, D.G., 1999. Compendium of Corn Diseases. American Phytopathological Socciety, St. Paul, MN, U.S.A.

Williams, L. D., Glenn, A. E., Zimeri, A. M., Bacon, C.W., Smith, M.A. and Riley, R. T. 2007. Fumonisin disruption of ceramide biosynthesis in maize roots and the effects on plant development and Fusarium verticillioidesinduced seedling disease. J. Agric.Food Chem. 55:2937-2946.

Wu, F. 2004. Mycotoxin risk assessment for the purpose of setting international regulatory standards. Environ. Sci. Technol. 38: 4049-4055.

Wu, F. 2006. Mycotoxin reduction in Bt corn: Potential economic, health, and regulatory impacts. Transgenic Res. 15:277-289.

Wu, J., and Miller, B. L. 1997. Aspergillus asexual reproduction and sexual reproduction are differentially affected by transcriptional and translational mechanisms regulating stunted gene expression. Mol. Cell. Biol. 17:6191-6201.

Yates, I. E., Arnold, J. W., Hinton, D. M., Basinger, W., and Walcott, R. R. 2003. Fusarium verticillioides induction of maize seed rot and its control Can. J. Bot. 81:422-428.

Zhao, Y., Su, H., Zhou, J., Feng, H., Zhang, K. Q., and Yang, J. 2015. The APSES family proteins in fungi: Characterizations, evolution and functions. Fungal Genet. Biol. 81:271-280. 